\title{
Una fortezza papale introversa trasfigura in uno spazio pubblico connesso. L'immagine della rocca Paolina di Perugia
}

\author{
Luca Martini
}

Abstract

Le rappresentazioni grafiche della rocca Paolina di Perugia appaiono sempre evocative del significato culturale che assume nella temperie in cui ogni immagine è ideata. Infatti i disegni architettonici del I 540 di Antonio da Sangallo il Giovane rappresentano un palazzo signorile che è anche una fortezza inespugnabile, mentre nelle vedute tra Seicento e Settecento il profilo della cittadella si confronta dimensionalmente con il tessuto urbano. Al contrario le prospettive ottocentesche, abbassando il punto di vista dell'osservatore, enfatizzano la monumentalità fuori-scala della rocca. E solo più di tre secoli dopo le planimetrie sangallesche, una planimetria progettuale del 1870 di Alessandro Arienti stravolge il ruolo della fortezza raccordandone i resti dei bastioni al nuovo centro cittadino postunitario con una rampa stradale, che sancisce la fine dell'imprendibilità della cittadella perugina che inizia così la sua trasformazione in spazio pubblico. E in tal senso si possono interpretare i disegni di progetto della risalita meccanizzata che nei primi anni '80 del Novecento ha permesso ai perugini e ai turisti di riappropriarsi definitivamente dei complessi locali voltati, oramai celebrati da fotografie geolocalizzate postate nei social network.

II testo propone una ricerca iconografica interpretando gli elaborati grafici che hanno come oggetto l'architettura perugina, dagli schizzi architettonici ai disegni fumettistici passando per le inquadrature cinematografiche, in quanto tali elaborati disvelano i ruoli assunti di volta in volta dalla macrostruttura paolina nel contesto urbano del capoluogo umbro, poiché smascherano una serie di sguardi tendenziosi su un'architettura che appare a tutt'oggi enigmatica.

Parole chiave

disegno della città, rocca Paolina di Perugia, immagini.

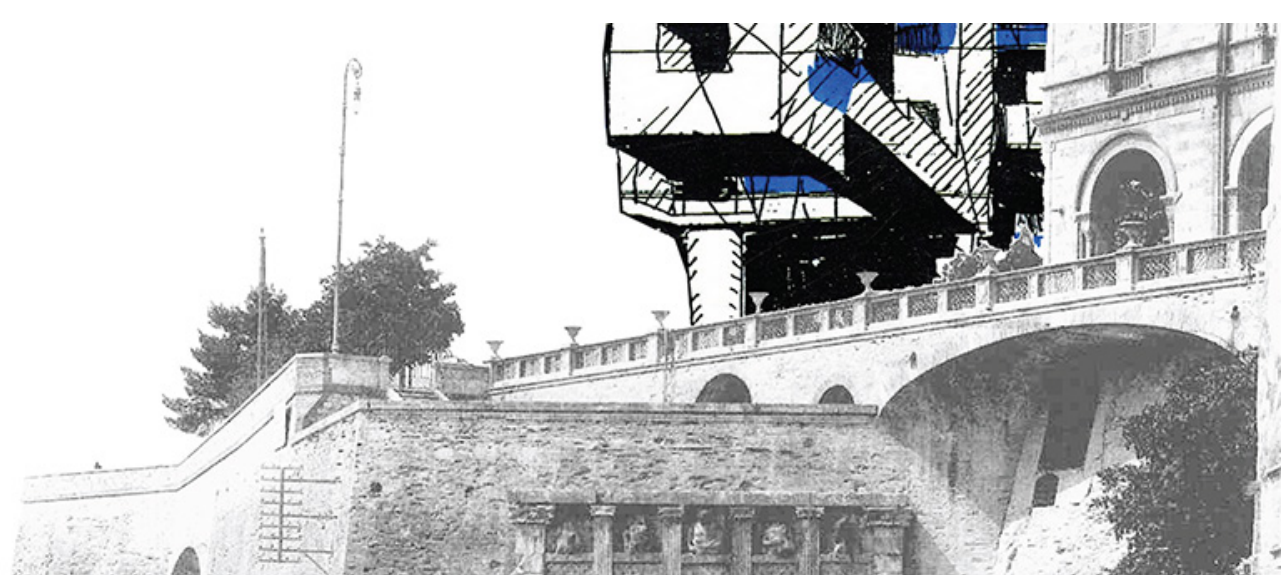


La rocca Paolina di Perugia è un'architettura enigmatica. II suo carattere sfuggente emerge ogniqualvolta gli studiosi ne tentano la comprensione, fin dal suo ruolo iniziale di fortezza militare giungendo alla sua funzione attuale di spazio pubblico. In questo senso la presente ricerca intende fornire una chiave interpretativa della rocca a partire dagli elaborati grafici che la rappresentano nei secoli, proponendo una lettura degli stessi volta a lumeggiare il background culturale dei loro autori. In modo da tentare una lettura diacronica per immagini del palinsesto architettonico paolino nelle vicende architettoniche del capoluogo umbro, attraverso un vero e proprio percorso di connessione visuale [Quici 20 I7].

I disegni di progetto degli anni ‘40 del Cinquecento di Antonio il Giovane e del suo entourage [I] rilevano puntualmente i lacerti etrusco-romani della città umbra, disvelando chiaramente le intenzioni progettuali inclusiviste dei Sangallo. Che però non tengono in debito conto la volontà papale, e infatti Paolo III ribalta il loro punto di vista minimizzando il ruolo del caposaldo difensivo verso la campagna ed enfatizzando il carattere militare della dimora verso la città [Belardi, Ceccucci 2006; Belardi, Martini 20 I5]. Laddove tale dimora viene edificata sopra le residenze della riottosa famiglia Baglioni, tanto da ricoprire un'intera porzione

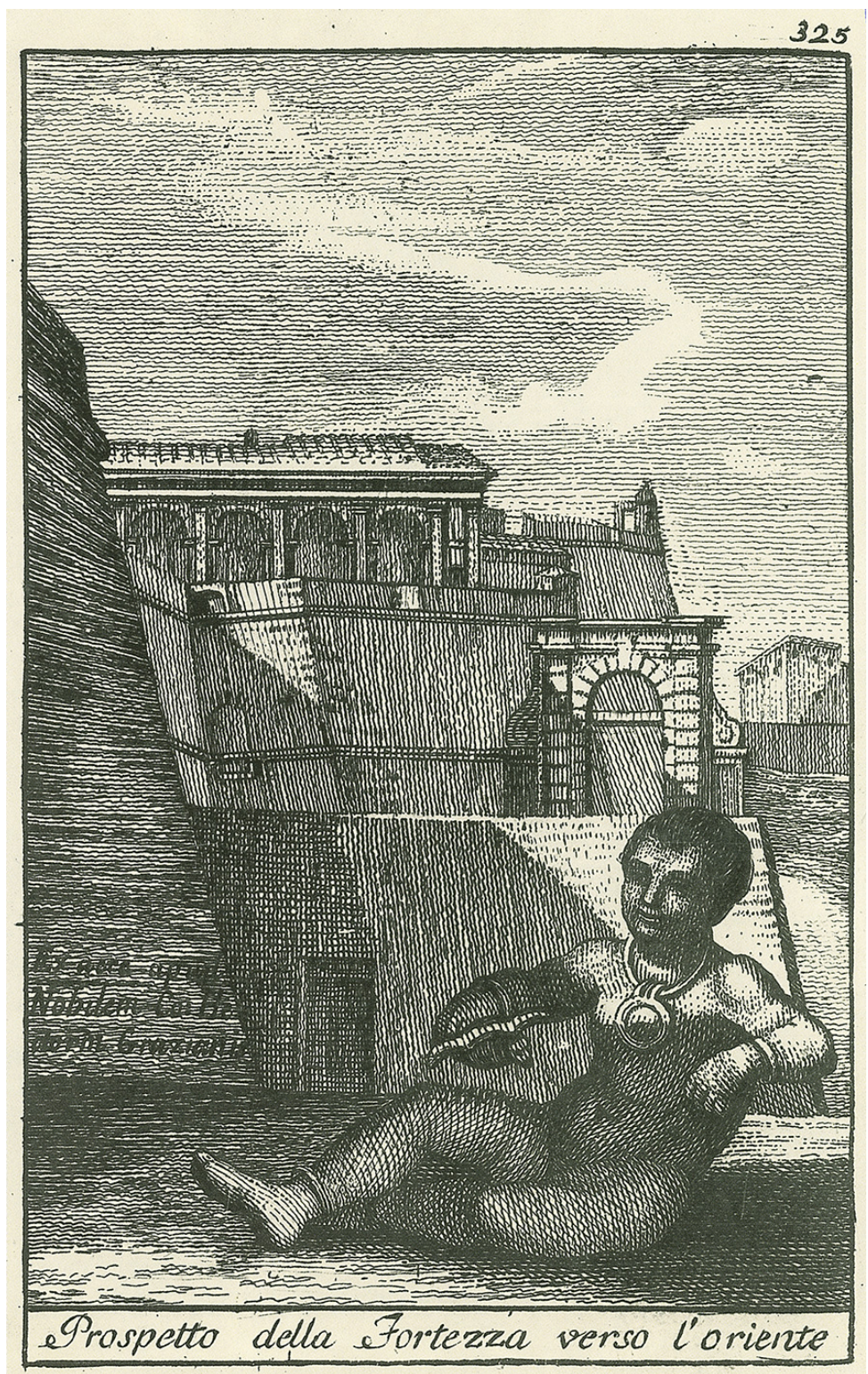


urbana con un terrapieno bordato da mura difensive che trasforma una città medievale in una caserma semi interrata, per cui i prospetti esterni dei caseggiati diventano quinte interne sovvertendo definitivamente l'immagine urbana. In questo senso i disegni di progetto raccontano la rocca secondo le intenzioni dei Sangallo, che però si allontanano ben presto dalla cura del cantiere [Camerieri, Palombaro 1992, pp. 9- I 8; Camerieri, Palombaro 1988, pp. 19-25]. Tanto da far pensare che l'esecutore del progetto paolino possa essere un giovane Galeazzo Alessi, che si forma in questo contesto in vista delle prime realizzazioni perugine che poi lo porteranno a consacrarsi a Genova e Milano [Belardi, Martini 20I5; Belardi 20 I3; Martini 20 I6, pp. 16, 59,60]. Non a caso, tra le porzioni più disegnate nel corso dei secoli, c'è proprio la loggia che Alessi progetta come ricercato punto di vista, che ad esempio è oggetto di una serie di esercitazioni ottocentesche in china e acquerello da parte degli studenti dell'Accademia di Belle Arti perugina. Che ne sottolineano il carattere duale dato dal rigore dell'ordine dorico e dalla plasticità del basamento rustico, resi in proiezioni ortogonali e prospettive frontali volte a interpretare il disegno del fronte [2].

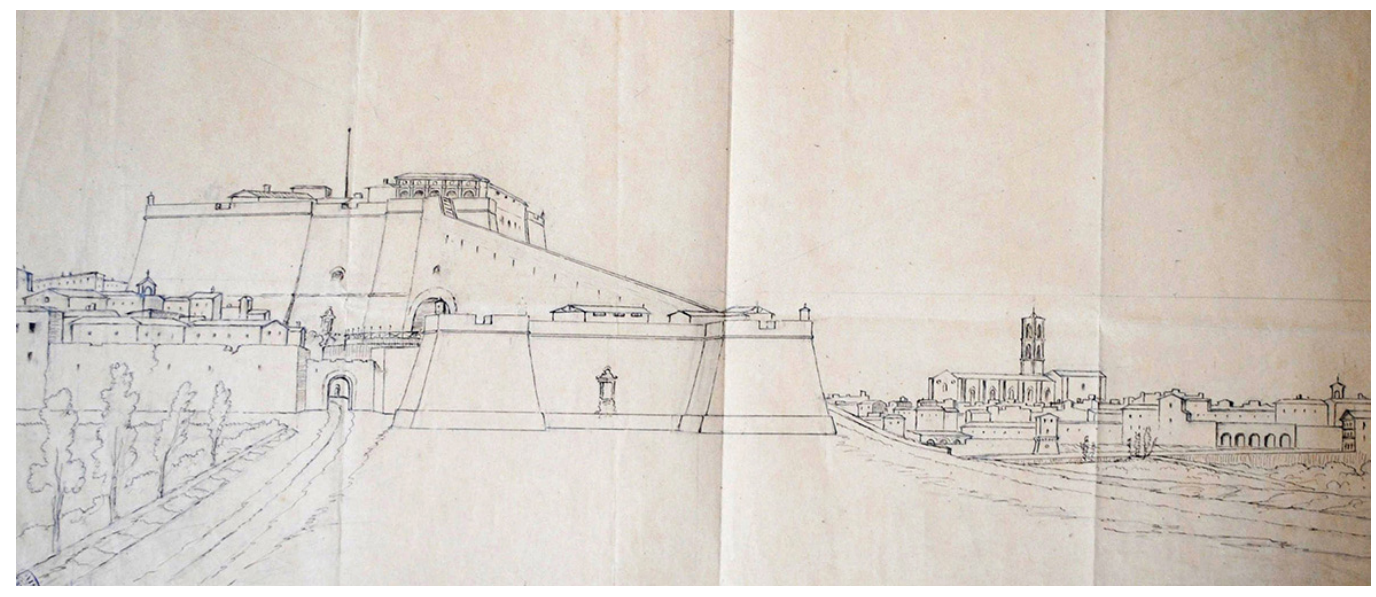

Negli anni la rocca Paolina vive un destino incruento, ma allo stesso tempo simbolizza il potere straniero sulla città che subisce inerte il dominio pontificio. E in questo senso nelle rappresentazioni tra Cinquecento e Settecento la mole della fortezza appare inserita placidamente nello skyline cittadino, in viste con un punto di osservazione molto distante dall'oggetto rappresentato, come le vedute a volo d'uccello, fino ad arrivare a un punto di vista improprio, come quello a distanza infinita delle planimetrie. Tali scelte proiettive sono declinate ad esempio nelle vedute di Georgis Hoefnagel (incisa nel I574) [3], di Cipriano Piccolpasso (I579) [4] e di Ignazio Danti (I58 I- I582) [5], o ancora nella prospettiva sempre di Hoefnagel incisa negli anni ' 40 del XVII secolo [6]. Anche nelle rappresentazioni planimetriche, come ad esempio la pianta rilevata da Giovanni Gambini del 1826 [7], sebbene sia misurabile lo scarto dimensionale tra l'impronta della rocca e quello delle altre architetture cittadine, spesso sia le scelte grafiche che l'ovvia mancanza di riscontri altimetrici determinano un sostanziale inserimento nel contesto urbano. La stessa concezione è espressa dal disegno di Baldassare Orsini Prospetto della Fortezza verso l'oriente, inciso da Raimondo Faucci nel 1784, che mette in scena il rapporto prolettico tra la loggia alessiana, la seicentesca porta San Carlo e una scultura bronzea etrusca in primo piano, celebrando la città papale al pari della città antica, ricordate entrambe nei secoli per la loro monumentalità difensiva [8]. Diversamente, nei decenni successivi, le rappresentazioni della rocca Paolina nel corso del XIX secolo manifestano un eclatante abbassamento del punto di vista volto ad amplificare il fuori-scala tra fortezza e misura urbana. Ad esempio, si assiste a questo fenomeno 
già dalla prospettiva del 1808 sempre di Orsini, ma in questo caso la rocca fronteggia gli edifici che la circondano grazie al punto di vista posto a una altezza comunque maggiore rispetto a quella umana [9]. Mentre la fortezza appare decisamente più minacciosa verso la città nel disegno di Giovanni Monotti degli anni 'I 0 dell'Ottocento in cui l'osservatore si abbassa ulteriormente, anche se il climax del disegno appare ancora quello di una placida veduta di una cittadina di provincia [10]. La stessa temperie si osserva nella celebre serie di dipinti a olio che Giuseppe Rossi dedica alla rocca tra il 1860 e il I 875 a partire da una serie di disegni precedenti [ I l]. Anche se la rappresentazione di entrambi i fortilizi uniti dal corridore inseriti nel contesto ne sanciscono in modo inequivocabile il rapporto fuori-scala con l'abitato, mettendo in scena quel sentimento popolare che avrebbe portato alla sua espulsione fisica dal tessuto urbano.

Al contempo, gli elaborati grafici costruttivi dei nuovi percorsi viari della città in trasformazione testimoniano l'annessione dell'area della rocca nella trama urbana [I 2]. Ma successivamente, il processo che porterà alla completa riappropriazione pubblica passa anche da una traumatica demolizione avviata nel I848, a seguito dei moti che portano alla fuga del papa da Roma e alla proclamazione della Repubblica Romana. Tale evento è immortalato da Nicola Benvenuti nell'opera La demolizione del forte paolino del I 850 circa, in cui la mole minacciosa rovina sotto i colpi di una folla festante. E la distruzione coincide figurativamente con la perdita irrimediabile della riconoscibilità urbana, che da quel momento è demandata alla riscoperta degli spazi ipogei adibiti fino ad allora a esclusivo uso militare, e che rimarranno pressoché misconosciuti per più di un secolo [13]. E se la libertà riconquistata dai perugini è solo temporanea poiché la restaurazione papale avviene già nel I849, l'immagine della fortezza non sarà più la stessa. Ad esempio, gli elaborati di rilievo dei resti e di progetto di una nuova caserma commissionati a diversi ufficiali del genio pontificio tra il I849 e il I856 documentano lo stato di rudere dell'area dell'ex piazza Rivarola [14], al pari di una ripresa fotografica della fine degli anni '50 del fotografo Robert Macpherson [15]. Tale condizione

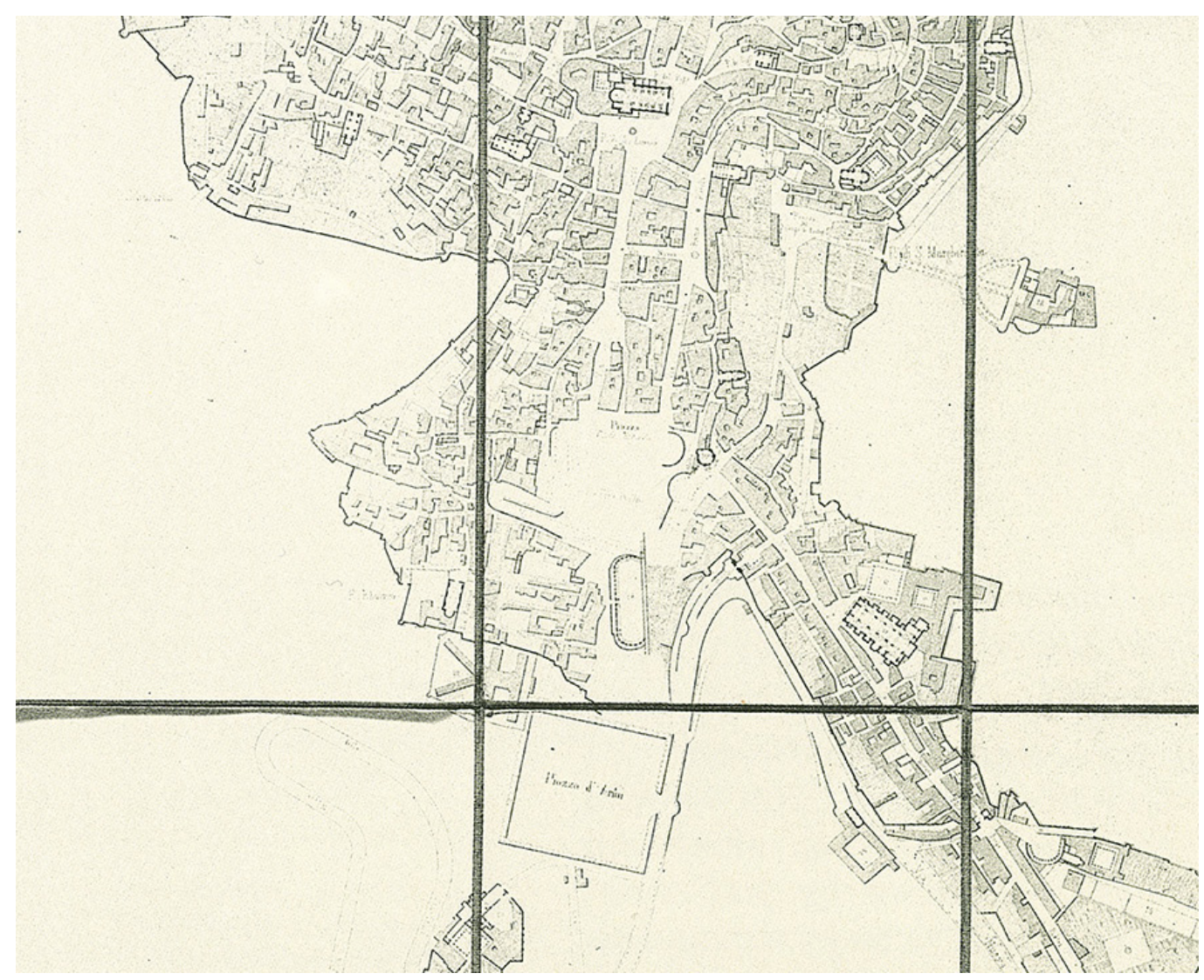


risulta anche da un gruppo di disegni e dipinti sempre della metà del diciannovesimo secolo del pittore Silvestro Massari conservati presso l'Accademia perugina, ma che ritraggono anche l'enigmatica loggia alessiana e gli interni chiaroscurali, prefigurandone la riconfigurazione e il riutilizzo, che purtroppo avverrà solo dei secondi [ [6]. Inoltre, una ripresa di quegli anni evidenzia la porta Marzia inglobata nelle murature sangallesche ma tamponata, a suggellare il disinteresse per i resti sotterranei [17]. Infatti, di lì a poco le immagini fotografiche testimoniano le prime realizzazioni superficiali sulle aree una volta occupate dai forti, come il teatro Politeama di Guglielmo Calderini [ 18]. Ma allo stesso tempo la pianta topografica incisa da Giulio Delicati nel 1866 mostra uno spazio vuoto in corrispondenza dell'impronta della rocca, ribadendone lo stato di vuoto urbano [19]. Infatti, i molteplici progetti che indagano lo spalto a ridosso del centro cittadino, sebbene coinvolgano progettisti illustri (da Nazareno Biscarini a Alessandro Arienti), non prevedono il riutilizzo delle parti interrate, le uniche sopravvissute del palinsesto fortificato, ma eliminano i ruderi superficiali e lasciano quale segno diruto solamente lo sperone seminterrato a sud-est [20]. Ma, a ben guardare, due disegni di Arienti del 1870 dichiarano il rapporto conoscitivo del nuovo palazzo della Provincia con gli ambienti sottostanti. In particolare, lo schema delle fondazioni e i dettagli dei rinforzi delle murature di sostegno testimoniano il legame fisico tra antica rocca ipogea e nuovo palazzo apogeo [2I]. Ma è soprattutto una planimetria progettuale sempre del 1870 e sempre di Arienti a stravolgere il ruolo della fortezza, laddove i resti dei bastioni che custodiscono mirabolanti spazi ipogei vengono raccordati al nuovo centro cittadino postunitario con una rampa della nuova strada realizzata contestualmente al palazzo pubblico, che mette ancora una volta in luce la presenza di una città sotterranea pressoché dimenticata [22]. E seppure negli anni in occasione dei passaggi di proprietà tra amministrazioni pubbliche siano redatti una serie di rilievi della porzione di rocca ipogea, nel periodo successivo continua l'oblio iconografico che corrisponde a un uso saltuario delle parti prive di macerie [23]. Allo stesso modo, anche le riprese fotografiche dei primi anni del Novecento che ritraggono la porta Marzia, seppur testimonino la riapertura della stessa, l'avvio del recupero e la condizione di stratificazione urbana, confermano implicitamente l'inaccessibilità dell'interno [24]. E se i documenti riportano che negli anni '30 inizia il recupero di parti dello spazio ipogeo [Lattaioli 1992, pp. 172-175], occorre aspettare il 1942 perché si ridesti l'interesse di ricerca attraverso un disegno ricostruttivo di Ugo Tarchi della rocca farnesiana [25]. Mentre nel

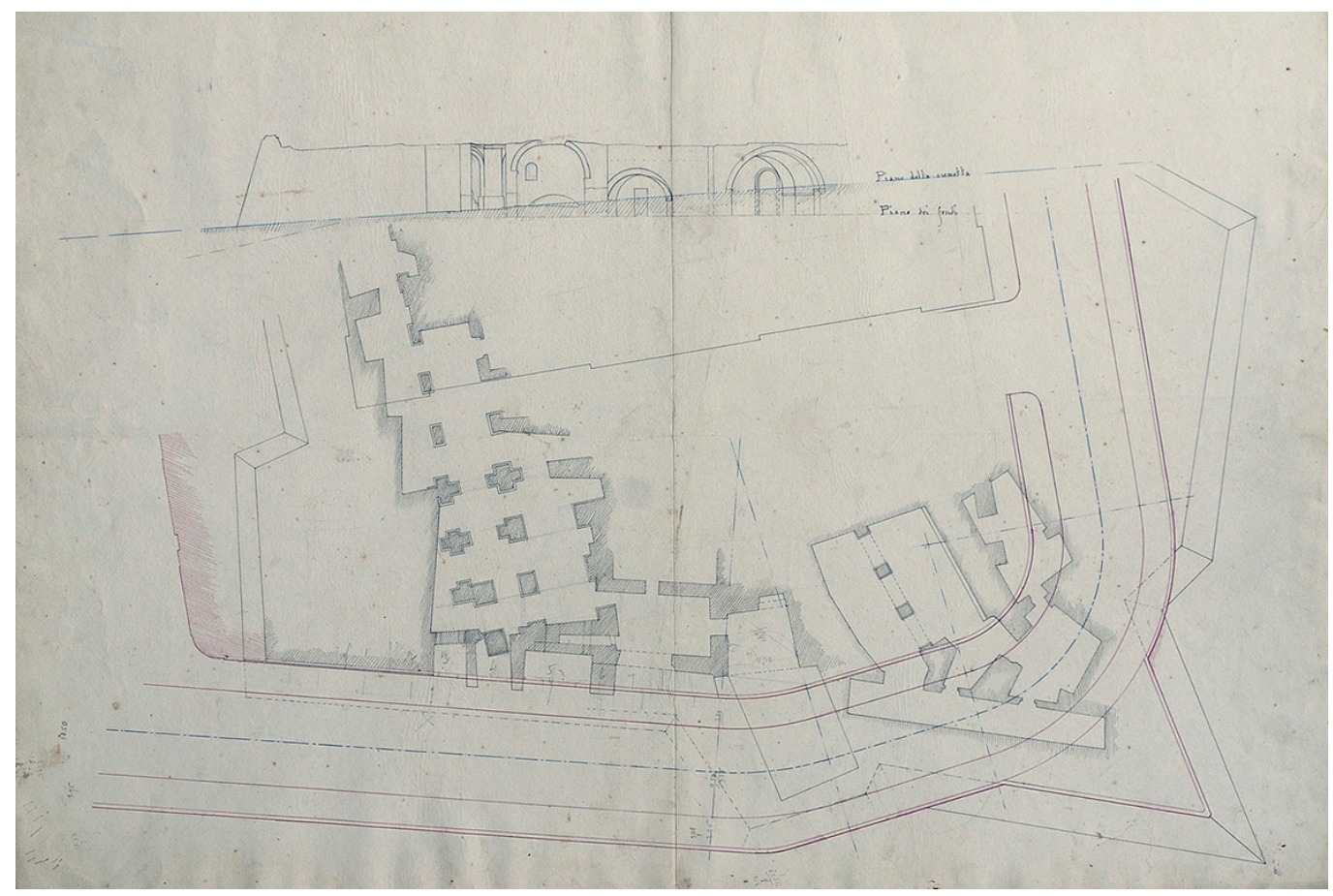


1943 viene riutilizzata come ricovero antiaereo per il palazzo governativo, come emerge da alcuni elaborati progettuali [26]. E soprattutto negli anni seguenti alcuni ambienti sono utilizzati a fini espositivi, ad esempio nel 1980 per l'iniziativa di rilevanza internazionale che vede protagonisti gli artisti Alberto Burri e Joseph Beuys, immortalata dal fotografo romano Lionello Fabbri [27].

Di lì a poco, il vero coup de théâtre che sancisce la riappropriazione pubblica della rocca avviene nel 1983 con la realizzazione del sistema di scale mobili che, attraversandola, unisce l'area di piazza Partigiani con l'acropoli del capoluogo umbro. Tale intervento è rappresentato negli elaborati grafici del 1979 redatti dall'équipe dell'Ufficio Tecnico del Comune di Perugia coordinata dall'ingegnere capo Mario Belardi, che sanciscono la definitiva riappropriazione degli spazi storici interrati. Che diventano così il contesto ritratto dai turisti nei selfie postati sui social network o nelle foto sferiche di Google Maps [28]. Successivamente,

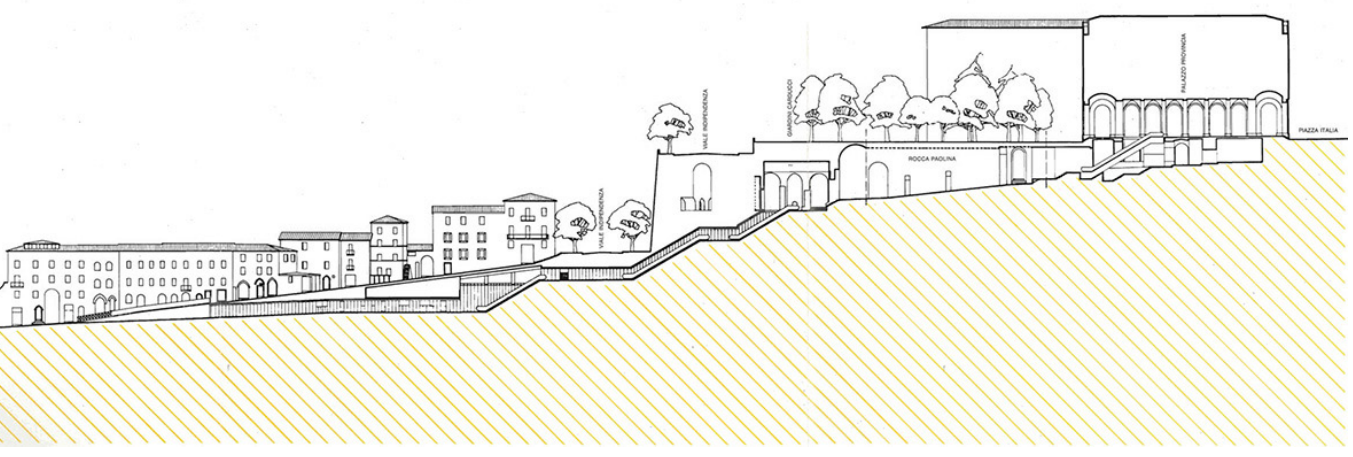

l'onda comunicativa che vede protagonista la rocca è conclamata dalla scelta della regista Liliana Cavani di eleggerla ad ambientazione medievale di alcune scene del lungometraggio Francesco del 1989, che proclama cinematograficamente i suoi percorsi chiaroscuri a emblema dell'età di mezzo. In questo senso, proprio in quegli anni prendono le mosse gli studi scientifici multidisciplinari sulla storia della rocca destinati a sfociare in volumi dedicati che accolgono disegni interpretativi e ipotesi ricostruttive [La Rocca Paolina 1992; Camerieri, Palombaro 1988], che si compiono idealmente nel 2009 con la pubblicazione del volume Il palazzo della provincia di Perugia [Mancini 2009]. Risalgono invece ai primi anni '90 le esercitazioni didattiche progettuali promosse da Adriana Soletti e Roberto de Rubertis, che in due occasioni pubbliche presentano gli elaborati grafici redatti dagli studenti volti ad approfondire l'accessibilità dell'area riannodandola alla trama urbana prefigurando un prolungamento insieme spaziale e tecnologico del sistema delle scale mobili [29]. Ugualmente, anche le riprese fotografiche che testimoniano le installazioni video ambientate da Fabrizio Plessi nel 1995 in occasione dell'esposizione La Rocca Elettronica connettono gli spazi della rocca alla contemporaneità [Schepers 1995; Lattaioli, Lattaioli 2004, pp. 108, 109]. Al pari di quelle che rappresentano il Museo della Città promosso dal Comune di Perugia e ideato da Italo Rota tra il 1999 e il 2003, in cui il vetro serigrafato, la grafica accattivante e le proiezioni comunicative si confrontano con le pietre centenarie [Belardi, Merli 2009, p. 8 I] . E tra i materiali esposti in quel contesto rimangono visibili ancora oggi i modelli plastici di resina polimerizzata della città medievale e rinascimentale realizzati da ONEOFF nel 2004 grazie alle nuove tecnologie di prototipazione rapida, a partire dalle elaborazioni digitali di Paolo Camerieri e Francesco Miniati. Inoltre gli stessi autori, con la collaborazione di Fabio Palombaro, sempre nel 2004 animano le vicende storiche della fortezza nel video La forma della memoria. La città ritrovata. La costruzione della Rocca Paolina di Perugia grazie alle possibilità ricostruttive della grafica tridimensionale [30]. D'altro canto, cinque anni dopo Daniele 
Giovagnoni e Marco Vergoni disegnano e sceneggiano le vicende storiche del monumento in un volume a fumetti che ne illustra in chiave divulgativa le potenzialità urbane latenti [Giovagnoni,Vergoni 2009] [3 I]. E infine anche il noto fotografo Steve McCurry ritrae nel 2012 gli spazi della rocca per la campagna di comunicazione Sensational Umbria promossa dall'amministrazione regionale, mettendoli in relazione figurativa con la manifestazione Umbria Jazz, che da decenni riscopre i luoghi del centro storico perugino in chiave di aggregazione sociale e culturale [Sensational Umbria 2014].

In effetti, a tutt'oggi la rocca appare un luogo onirico tra passato e futuro, quasi un eterno 'non finito' solo in parte inserito nell'intreccio urbano perugino, come un palinsesto per certi versi ancora interrotto [32]. In questo senso gli studenti dell'insegnamento di Progettazione digitale del Corso di Laurea in Ingegneria edile-Architettura dell'Università degli Studi di Perugia hanno affrontato l'esercitazione progettuale danteum. oggi nell'anno accademico 20।2-2013 attraverso l'ideazione di percorsi fisici di conoscenza di sé tra le profondità sotterranee e l'immensità del cielo', utilizzando i linguaggi della composizione e della rappresentazione architettoniche. Ad esempio, il progetto tensioni di luce amplifica i multiformi spazi ipogei in uno stereometrico giardino apogeo posto iconicamente sopra il palazzo della Provincia, raccordandoli attraverso una rampa ascensionale che, grazie alle tecniche contemporanee di disegno digitale, reinterpreta i mondi danteschi e l'esperienza progettuale di Giuseppe Terragni [33]. Infine, il rilievo architettonico redatto da Giulia Bazzucchi nell'anno accademico 2016-2017 in occasione della tesi di laurea magistrale in Ingegneria edile-Architettura riannoda il filo che unisce la città e la rocca in quanto, per la prima volta, le piante e le sezioni degli spazi sono ambientate nel contesto risignificando in chiave urbana il dedalo paolino [Bazzucchi 2016-2017]. E non è un caso se è proprio un disegno 'in punta di mouse' che analizza gli ambienti in chiave cognitiva, poiché il disegno è lo strumento speculativo e prefigurativo per antonomasia. E in questo senso la parabola visionaria di una 'rocca rilevata' che diventa una 'rocca progettata' è quanto mai auspicabile, in quanto 'nuovi sguardi' e 'nuove immagini' rappresentano il futuro percorribile della città contemporanea [34]. Anche a Perugia.

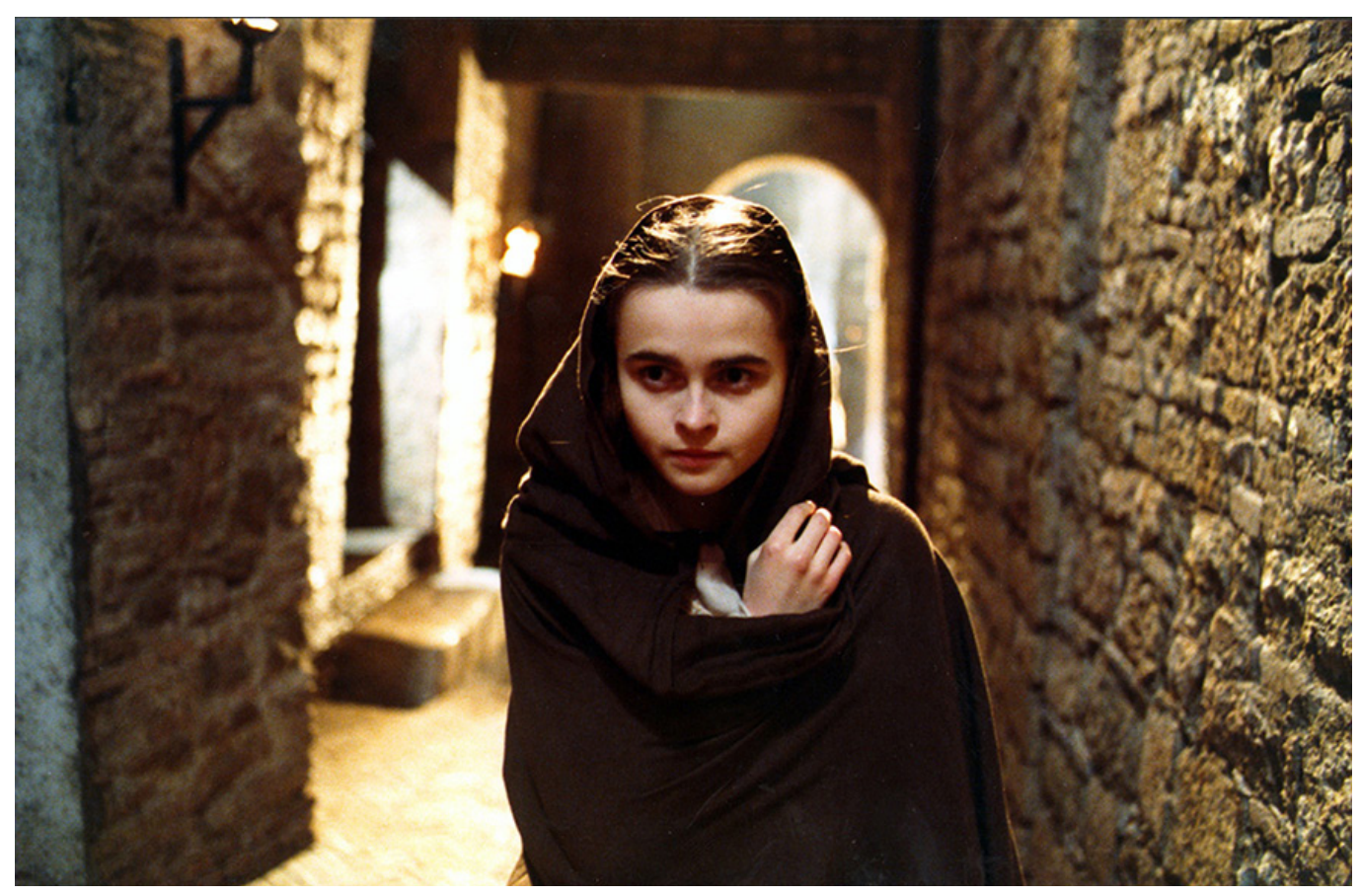




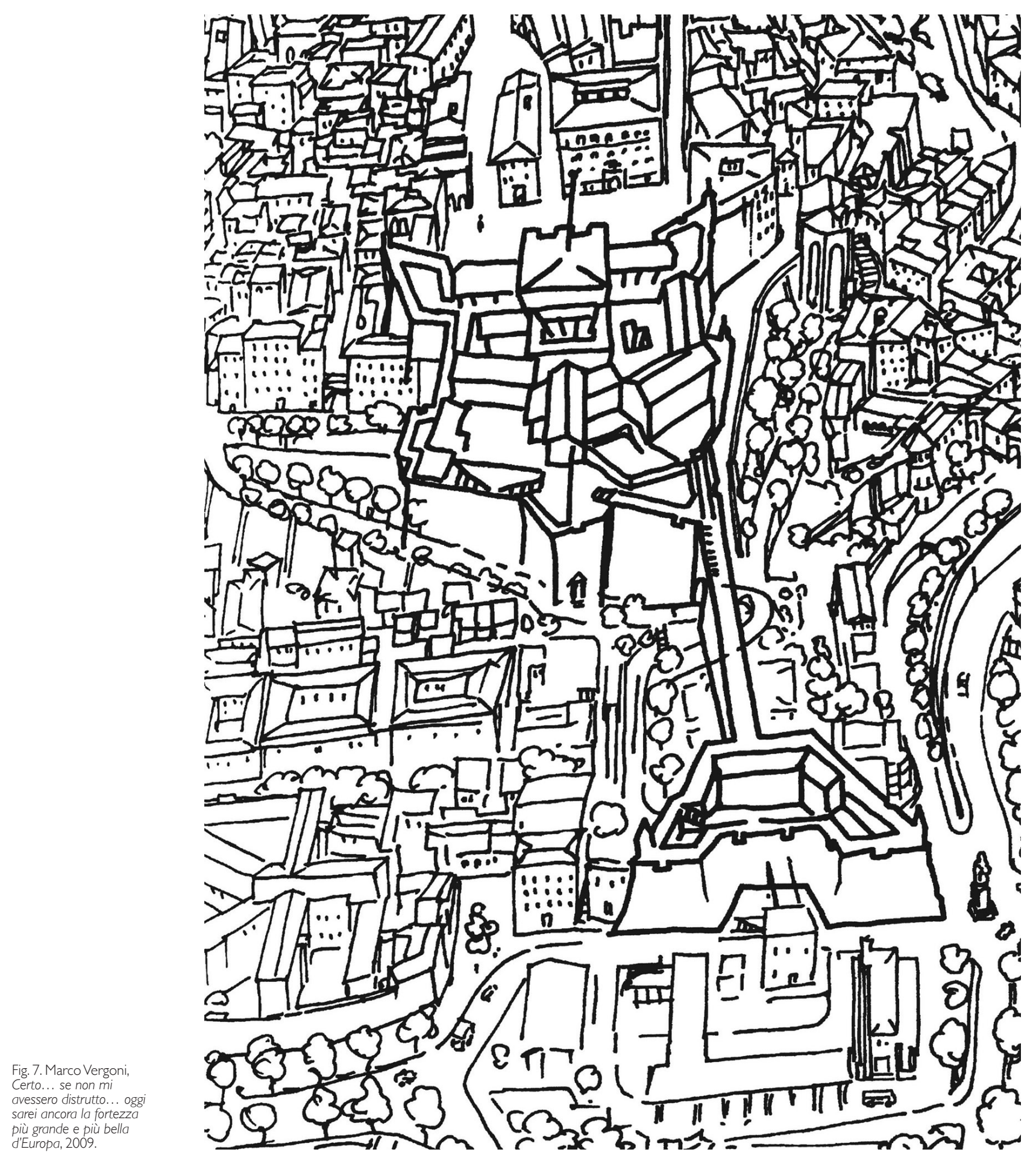



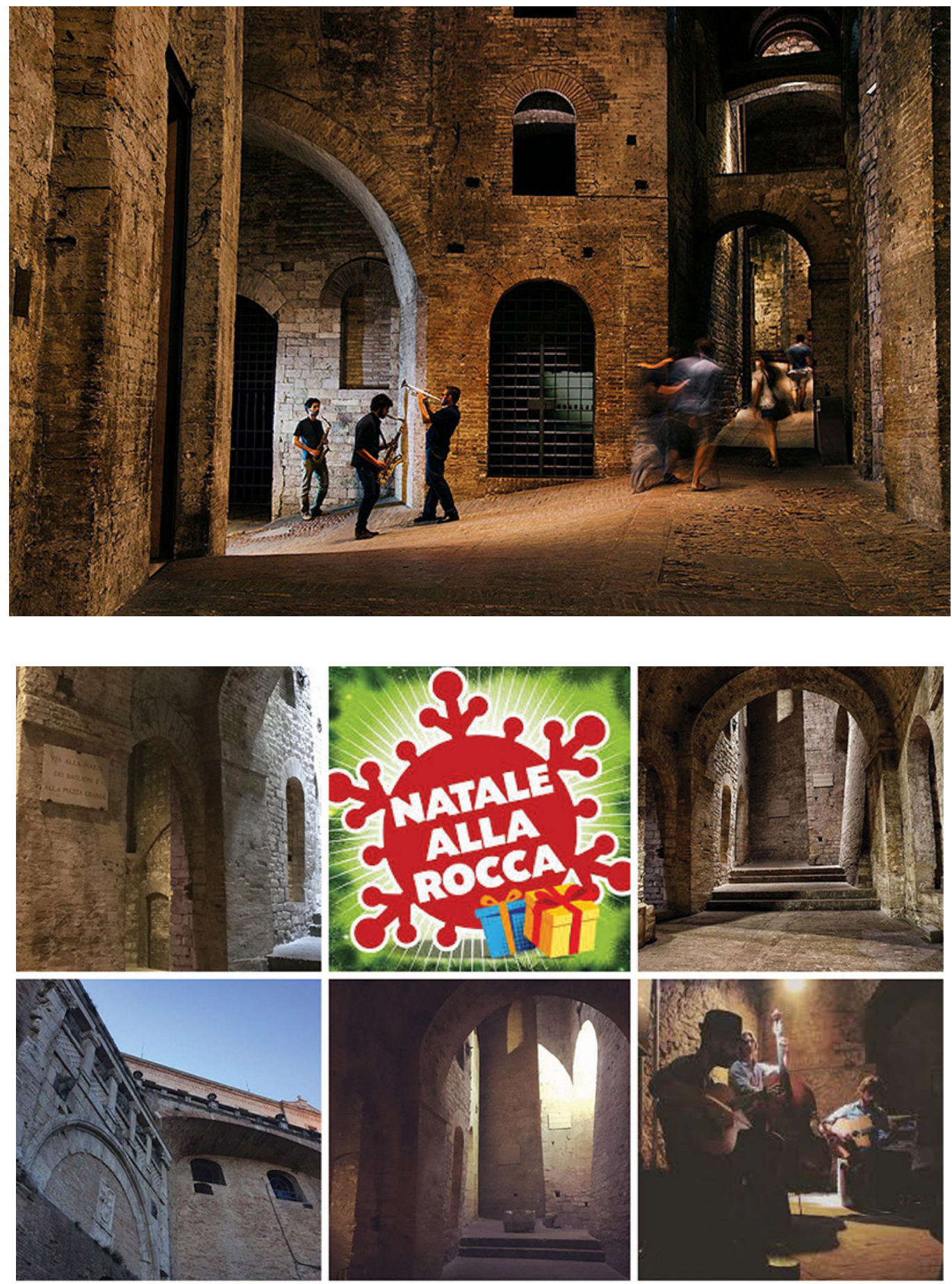

Fig. 9. \#roccapaolina
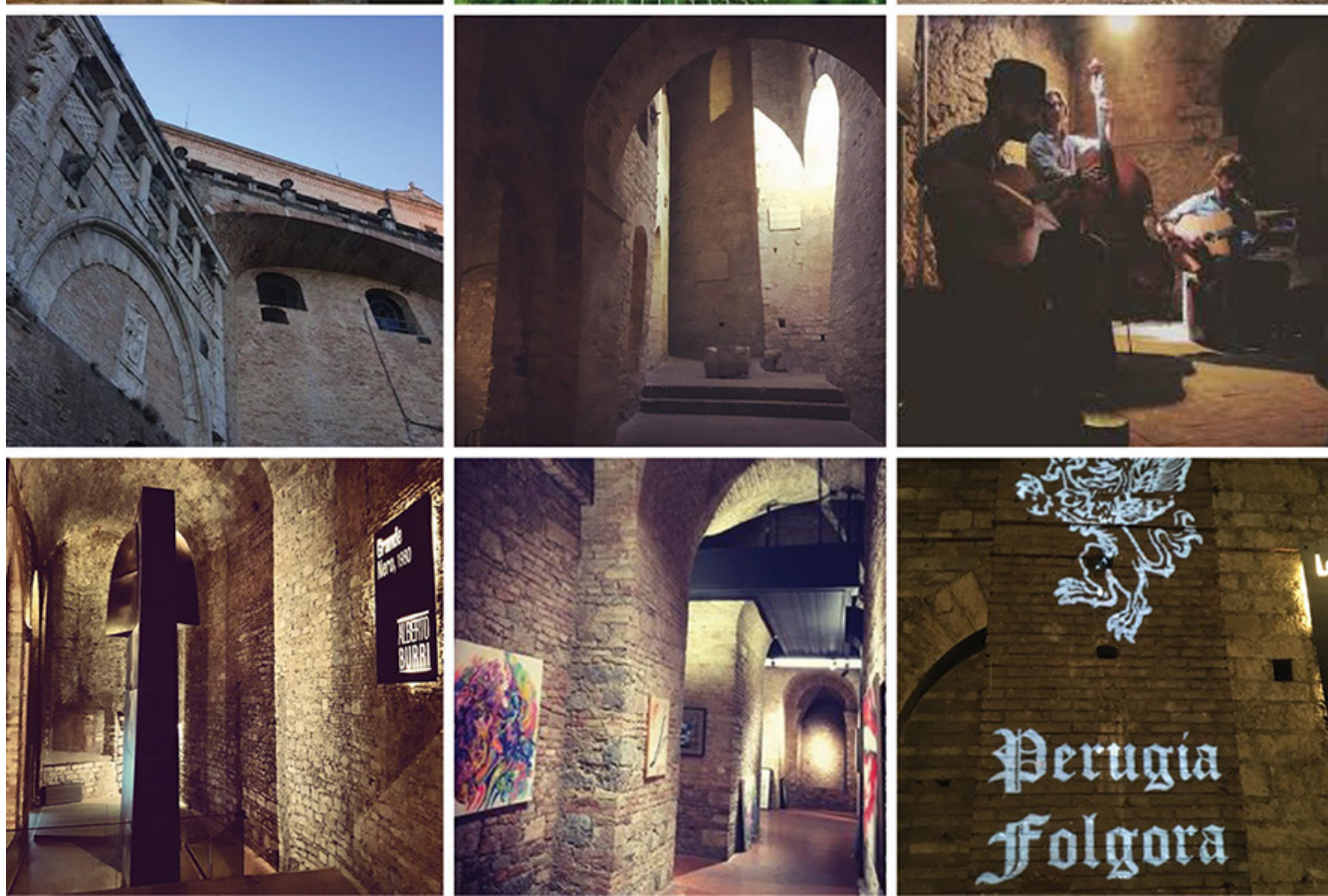
Fig. 10. Luca Martini, Città spaziale. Perugia e locandina dell'iniziativa danteum. oggi, 2013

Fig | | . Marta Berrettoni Elisa Bettollini, Eugenio Bini, Marco Calderini, Malvina Maria Manfroni, Simone Moroni, Daniele Ripa, tensioni di luce, 2013; sezione.

Fig. 12. Giulia Bazzucchi, Nuova luce sulla rocca.

Rilievo architettonico della Rocca Paolina di Perugia e progetto illuminotecnico della Casa di Gentile Baglioni, 20 17; sezione.
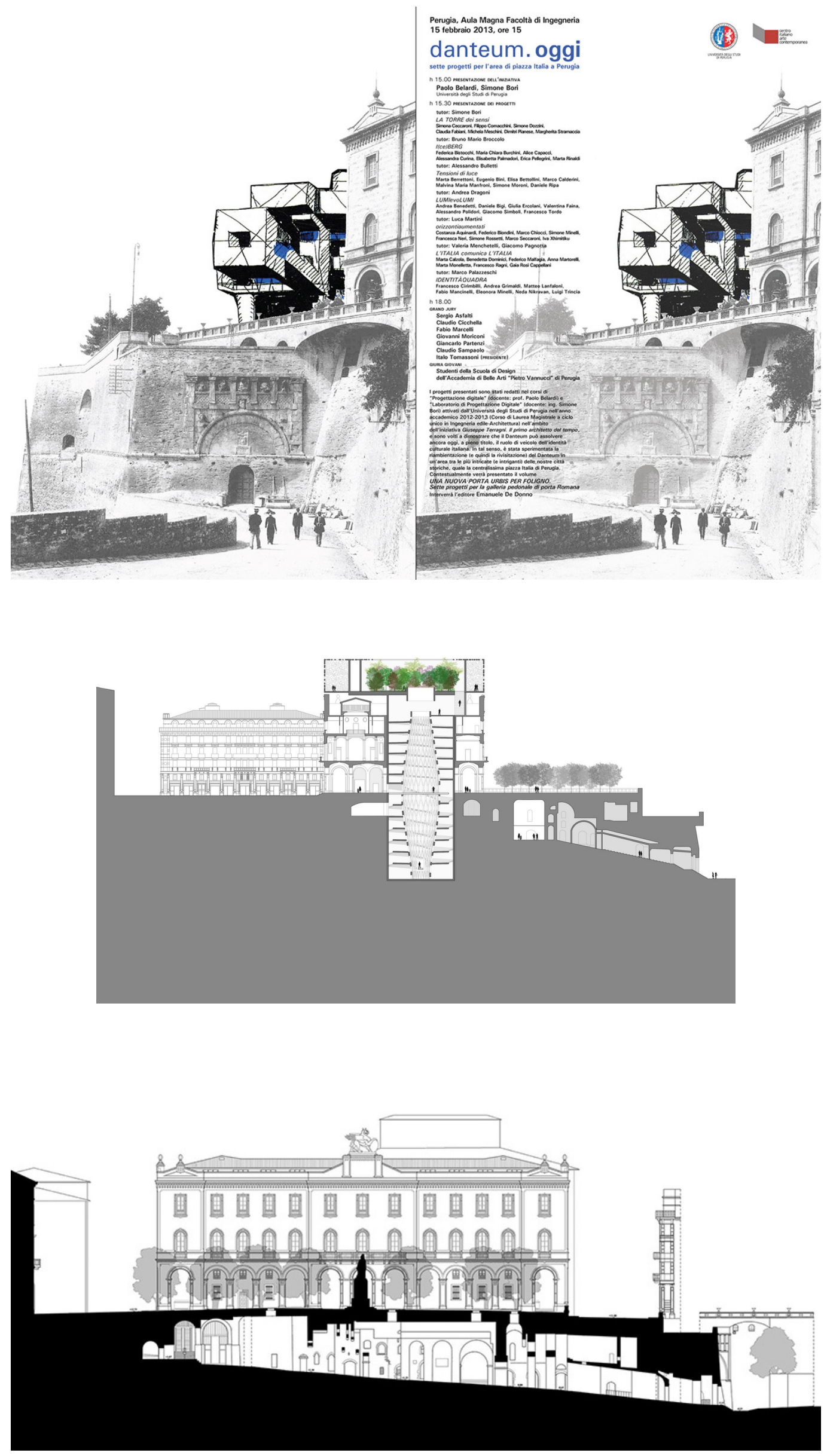


\section{Note}

[I] I disegni dei Sangallo sono conservati presso il Gabinetto dei Disegni e delle Stampe della Galleria degli Uffizi di Firenze [La Rocca Paolina 1992, pp. 2 I, 23, 28, 29, 33, 47, 5 I, I I0, I I 2, I I 5, I I6, I I 8; Camerieri, Palombaro I 988, pp. 90, I 27- I 50].

[2] Gli elaborati sono conservati presso l'archivio dell'Accademia di Belle Arti "Pietro Vannucci" di Perugia. Si veda: Odoardo Biagini, Pianta e alzato della loggia Alessi, I883; Lorenzo Carloni, Alzato e pianta della loggia dell'Alessi, I883. Inoltre, un altro disegno è conservato presso la Galleria Nazionale dell'Umbria: Stanislao Nicolai, Portico dell'Alessi situato nel Forte, I824. Si veda anche il progetto di due fontane in piazza della fortezza conservato sempre presso l'Accademia di Perugia: Napoleone Cherubini, Pianta Topografica di piazza della fortezza, 1834.

[3] Franz Hoogembergh, Perusia. Gratum musis in Tuscia domicilium, I 574 [Cassano 1990, I, pp. 202, 203].

[4] Cipriano Piccolpasso, II primo libro delle Piante et ritratti delle Citta e Terre dell'Umbria, I579, f. XXXIII (manoscritto 3064 conservato presso la biblioteca Augusta di Perugia) [Cecchini 1963].

[5] La veduta di Danti è affrescata presso la galleria del Belvedere nei Musei Vaticani: Ignazio Danti, Perusiae Augustae. Corographia, I58I-I582 [Matteini Chiari, Caponi 20I0, p. 38].

[6] Matthäus Merian, Perusia, 1640 [Cassano 1990, II, pp. 220, 22 I].

[7] Giuseppe Canacci, Pianta della Città di Perugia, I 826 [Cassano 1990, I, pp. 250, 25 I].

[8] Raimondo Faucci, Prospetto della Fortezza verso l'Oriente, I 784 [Cassano 1990, II, pp. I4, I5; Belardi 20 I9].

[9] Raimondo Faucci, Prospetto della Piazza Nuova dinanzi alla Fortezza, con le Fabbriche che sono situate attorno..., 1808 [Cassano 1990, II, pp. 40, 4I].

[10] Giuseppe Carattoli, Frezzolini, Prospetto della Piazza Napoleone e Fortezza in Perugia, I 810 [Cassano 1990, II, pp. 68, 69] Si veda anche la prospettiva di Annibale Angelini:Annibale Angelini, Napoleone Cherubini, Piazza di Minerva una volta Collegio Pio disegnata come se avesse avuto il suo compimento, I 835 [Cassano 1990, II, pp. 90, 9I].

[I I] I disegni di Rossi sono conservati presso l'archivio dell'Accademia di Belle Arti "Pietro Vannucci" di Perugia e i suoi dipinti presso la Galleria Nazionale dell'Umbria. Si veda: Giuseppe Rossi, Veduta del fianco occidentale della Fortezza Paolina, 1870 [Belardi, Bori, Menchetelli 20 I0, p. 66; Guerrini 1992, pp. I22, I23; Lattaioli 1992, pp. I 38, I 39, I45].

[12] Ad esempio la strada che unisce l'area della porta cosiddetta dei Tre Archi con l'attuale piazza Italia, disegnata nel I829 dall'ingegnere pontificio Giovan Battista Cerrini. I relativi disegni tecnici sono conservati presso l'Archivio di Stato di Roma [Lattaioli 1992, pp. |4|-|43].

[13] L'opera di Benvenuti è conservata in una collezione privata. Si veda: Nicola Benvenuti, La demolizione del forte paolino, 1850 [Belardi, Bori, Menchetelli 20 I0, p. 66; ]. Si veda anche: Annibale Brugnoli, Benedetto Baglioni si reca ad abbattere il Forte Paolino, 1890 [Lattaioli, Lattaioli 2004, p. 77]

[14] Ad esempio gli elaborati redatti da Costantino Forti sono conservati presso la Soprintendenza Archeologia, Belle Arti e Paesaggio dell'Umbria e l'Archivio di Stato di Roma [Banti, Ercolani 1992, pp. 195- I98, 202-206, 208-209; Banti, Ercolani 1992. pp. 199-20I].

[15] Una stampa della ripresa di Macpherson è conservata presso l'archivio della Biblioteca dell'Università di Glasgow. Si veda Robert Macpherson, Porta etrusca di Perugia, 1858 [Trinei 2016, p. 28].

[16] I disegni e i dipinti di Silvestro Massari sono conservati presso l'archivio dell'Accademia di Belle Arti "Pietro Vannucci" di Perugia [Belardi, Merli 2009, p. 58].

[17] La ripresa fotografica di autore ignoto è conservata in una collezione privata. Si veda: Veduta della porta Marzia murata, 1865 [Bozzi 1992, p. 226].

[18] Alessandro Baldeschi Eugenj, Veduta di Perugia dall'educatorio di Sant'Anna, I865 [Trinei 20I6, p. 35]; Alessandro Baldeschi Eugenj, Veduta di Perugia dal campanile di Santa Giuliana, 1870 [Trinei 20 I 6, p. 35]; Cesare Polozzi, Veduta di Perugia dal campanile di San Pietro, 1880 [Trinei 2016, p. 164]. Le riprese citate sono conservate in una collezione privata.

[19] Giulio Delicati, Pianta topografica della città di Perugia, I 866 [Cassano 1990, I, pp. 274, 275].

[20] Ad esempio, nella proposta progettuale per il concorso pubblico del 1863 di Nazareno Biscarini e Americo Calderini, conservata presso l'archivio dell'Accademia di Perugia, gli spazi ipogei non sono coinvolti dal progetto seppure disegnati. Si veda: Nazareno Biscarini, Americo Calderini, Prospetto, I863 [Neri 2009, p. 95].

[2 I I I disegni di progetto di Alessandro Arienti sono conservati presso l'archivio della Provincia di Perugia. Si veda: Alessandro Arienti, Pianta delle fondazioni, I 870 [Neri 2009, p. I05].

[22] Alessandro Arienti, Tracciato stradale di viale Indipendenza, I870 [Belardi, Merli 2009, p. 70].

[23] Ad esempio, i rilievi del palazzo della Provincia sono conservati presso l'Archivio Notarile Distrettuale di Perugia: Pianta dei fondi, 1874 [Tosti, Mori 2009, p. 162].

[24] Fratelli Alinari, Porta Marzia, I 890 [Trinei 20 I6, p. 204]; Fratelli Alinari, Porta Marzia, 1920 [Bozzi I992, p. 247]. Entrambe le riprese sono conservate presso gli archivi Alinari a Firenze.

[25] Ugo Tarchi, Porta Marzia, 1942 [Tarchi 1942, t. LXXX]. 
[26] II progetto del ricovero antiaereo del 1943 è conservato presso l'Archivio di Stato di Perugia: Profilo longitudinale del nuovo braccio di galleria, 1943 [Tosti, Mori 2009, p. 188].

[27] Tale incontro lascia tracce anche nella Perugia contemporanea poiché le lavagne su cui Beuys disegna la sua lezione sull'arte sono attualmente esposte presso il Museo Civico di Palazzo della Penna, mentre a seguito di tale iniziativa la scultura Grande Nero di Burri è tuttora esposta presso lo sbarco delle scale mobili della rocca [Tomassoni 1980; Tomassoni 2003, pp. $22,25,38]$.

[28] Mobilità 1985. Si veda anche: Ufficio Tecnico del Comune di Perugia, Percorso pedonale meccanizzato tra il parcheggio pluripiano di Piazza Partigiani e Piazza Italia, 1979 [Belardi, Merli 2009, p. 72; Lattaioli 1992, pp. 176, 177].

[29] Si veda: Disegnare Perugia 1992 e Proposta di sistemazione 1993, pp. I I5-121. Si veda anche: Roberto de Rubertis, Proposta per la sistemazione di Aliante sul bastione meridionale della Rocca Paolina, 1995 [Belardi, Merli 2009, pp. 80, 8I] Roberto de Rubertis, Perugia.Tracciato delle mura etrusche, 1983 [de Rubertis 20 I I, p. I7]. Si veda anche Cancellaro $1994-1995$.

[30] Si veda l'animazione video online: <youtube.com/watch?v=LHrxTb IRGas> (26 febbraio 2020).

[3।] Quella citata non è l'unica occasione in cui la rocca è disegnata in un racconto a fumetti; infatti, nel 1980 Moreno Chiacchiera rappresenta la rocca in costruzione nel volume Due o tre cose che sappiamo della nostra città... [Chiacchiera, Peverini,Todini 20 17, p. 49] e nel 1983 il fumettista francese Gilles Chaillet rappresenta un'ambientazione esterna della Perugia medievale evidentemente inspirata agli interni della rocca nel primo numero della serie Vasco, L'or et le fer [Chaillet I983, I].

[32] Nel novembre 20 I 4 la rocca Paolina è stata protagonista del seminario Ambienti, Stanze e altri Spazi Sonori. Interscalarità e Multisensorialità nella Rocca Paolina di Perugia, responsabile scientifico professoressa Alessandra Capanna, nell'ambito delle attività del Dottorato di Ricerca in Architettura, Teorie e Progetto del Dipartimento di Architettura e Progetto, della Sapienza Università di Roma. Si veda: Anna Riciputo, Wired Voices | Vocifilate. Lo spazio delle installazioni sonor:: <utopiaspa.wordpress. com/20 I 7/ I/05/istallazione-wiredvoices> (26 febbraio 2020). Inoltre, la rocca Paolina ospita dal 2017 la videoinstallazione immersiva Perugia Folgora che, seppur negli spazi limitati di una singola sala, tenta di riconnettere poeticamente per immagini proiettate animate alcune fasi della storia della città con le pareti e le volte cinquecentesche. La videoinstallazione è stata prodotta da Daring House con la direzione creativa di Stefano Casertano e Monica Manganelli.

[33] Docenti dell'insegnamento Paolo Belardi e Simone Bori, tutor Simone Bori, Bruno Mario Broccolo, Alessandro Bulletti, Andrea Dragoni, Luca Martini, Valeria Menchetelli, Giacomo Pagnotta, Marco Palazzeschi. In tale ambito, il concept progettuale tensioni di luce è redatto dagli studenti Marta Berrettoni, Elisa Bettollini, Eugenio Bini, Marco Calderini, Malvina Maria Manfroni, Simone Moroni, Daniele Ripa. In particolare, la locandina che divulga la presentazione pubblica è redatta componendo graficamente una ripresa fotografica storica che rappresenta la porta Marzia, le mura sangallesche e il palazzo della Provincia con il disegno Città spaziale. Shanghai del 2002 di Yona Friedman, al fine di enfatizzare il carattere di stratificazione dell'area unito alla sua potenzialità di palinsesto urbano in continua trasformazione.

[34] Tali tematiche sono approfondite anche nell'ambito della presentazione pubblica Indagini scientifiche e proposte progettuali per un approfondimento delle conoscenze sulla rocca Paolina tenuta da Paolo Belardi il 12 maggio 2017 in occasione dell'iniziativa La Rocca Paolina. Una storia legata al sale a cura della Associazione Rione di Porta Eburnea di Perugia. Si veda anche: Belardi, Martini, Menchetelli 2020.

\section{Riferimenti bibliografici}

Banti Alessandro, Ercolani Riccardo (1992). Le trasformazioni dell'area della fortezza di Perugia nel periodo della formazione dello Stato unitario. In La Rocca Paolina di Perugia. Studi e ricerche. Perugia: Electa Editori Umbri, pp. 193-223.

Bazzucchi Giulia (20 I 6-20 I 7). Nuova luce sulla rocca. Rilievo architettonico della Rocca Paolina di Perugia e progetto illuminotecnico della Casa di Gentile Baglioni. Tesi di laurea, corso di Laurea Magistrale a ciclo unico in Ingegneria edile-Architettura, relatore prof. Paolo Belardi. Università degli Studi di Perugia.

Belardi Paolo, Martini Luca, Menchetelli Valeria (2020). La rocca Paolina di Perugia. Da baluardo dell'inaccessibilità a landmark dell'accessibilità. In Navarro Palazón Julio, García-Pulido Luis José (ed.). Defensive Architecture of the Mediterranean. Vol. XII. Granada: Universidad de Granada, Universitat Politècnica de València, Patronato de la Alhambra y Generalife, pp. I053- 1059.

Belardi Paolo (2019). "Ante litteram" hyper-drawings. Marginal notes on the application of proleptic abstraction in the history of art and architecture. In IMG journal, I (Manifesto), pp. 58-67.

Belardi Paolo (20 I3). Sui “disegni regolatori” di Galeazzo Alessi. In L'ingegnere umbro, I, p. I I.

Belardi Paolo, Bori Simone, Menchetelli Valeria (20 I 0). II palazzo nell'iconografia storica. II terzo prospetto. In Mancini Francesco Federico (a cura di). Il Palazzo Donini di Perugia. Perugia: Quattroemme, pp. 63-77.

Belardi Paolo, Ceccucci Filippo (2006). Ad coercendam Perusinorum audaciam. Galeazzo Alessi e la trasformazione di Perugia in "città farnesiana": considerazioni e ipotesi. In Robotti Ciro (a cura di). Dai Farnese ai Borbone. Famiglie europee. Costruire Stati. Lecce: Edizioni del Grifo, pp. I33-142.

Belardi Paolo, Martini Luca (20 I5). The Alessi measure in Perugia. In Disegnarecon, I5, pp. 19. I - 19.22.

Belardi Paolo, Merli Sonia (2009). La piazza. Da spalto fortificato a cerniera immateriale. In Mancini Francesco Federico (a cura di). Il Palazzo della Provincia di Perugia. Perugia: Quattroemme, pp. 53-89.

Bozzi Franco (1992). La distruzione della Rocca. In La Rocca Paolina di Perugia. Studi e ricerche. Perugia: Electa Editori Umbri, pp. 225-25I.

Camerieri Paolo, Palombaro Fabio (1992). La Rocca Paolina: dal "palazzo" alla "cittadella". Dal Sangallo a un "modo architettonico" comune. In La Rocca Paolina di Perugia. Studi e ricerche. Perugia: Electa Editori Umbri, pp. 9-I8. 
Camerieri Paolo, Palombaro Fabio (1988). La «Rocca Paolina» un falso d'autore. Dal mancato compimento alla radicale alterazione del progetto di Antonio da Sangallo il Giovane per il Forte di S. Cataldo. Perugia: Provincia di Perugia.

Cancellaro Pierpaolo (1994-1995). Rocca Paolina. Un progetto ipogeo per Perugia. Tesi di laurea, corso di Laurea in Architettura, relatore prof. Arrigo Rudi. Istituto Universitario di Architettura di Venezia.

Cassano Francesca Romana (1990). Perugia e il suo territorio. Incisioni dal XV al XIX secolo. Perugia:Volumnia.

Cecchini Giovanni (1963). Perugia del '500 nelle testimonianze di un artista. Cipriano Piccolpasso. Perugia: Sezione arti grafiche Istituto statale d'arte.

Chaillet Gilles (1983). Vasco. L'or et le fer. Une histoire du journal Tintin. Bruxelles, Paris: Editions Du Lombard, 1983.

Chiacchiera Moreno, Peverini Aldo, Todini Antonio (2017). Storia di Perugia. Due o tre cose che sappiamo della nostra città... Perugia: Edizioni Era Nuova.

de Rubertis Roberto (20I I). Rilievi archeologici in Umbria. Perugia, Assisi, Orvieto, Otricoli, Spoleto. Napoli: Edizioni scientifiche e artistiche.

Giovagnoni Daniele, Vergoni Marco (2009). La Rocca Paolina. Autobiografia di una fortezza. Perugia: Era Nuova.

Guerrini Giuseppe (1992). La Rocca Paolina: cittadella come microcosmo guerresco e sede di istituzioni nel secolo della sua costruzione. In La Rocca Paolina di Perugia. Studi e ricerche. Perugia. Electa Editori Umbri, pp. I09-I25.

Disegnare Perugia. "La piazza scopre la Rocca” (1992). Perugia: Galeno.

Lattaioli Paolo (1992). Effetti indotti dalla costruzione della Rocca Paolina sul tessuto urbanistico di Perugia. In La Rocca Paolina di Perugia. Studi e ricerche. Perugia: Electa Editori Umbri, pp. $133-192$.

Lattaioli Paolo, Lattaioli Marta (2004). La Rocca Paolina in Perugia. Visita e storia. Perugia: EFFE Fabrizio Fabbri.

Mancini Francesco Federico (a cura di). (2009). Il Palazzo della Provincia di Perugia. Perugia: Quattroemme.

Martini Luca (2016). Alessi disegnato. Rilievo architettonico delle opere umbre di Galeazzo Alessi. Perugia: Fabrizio Fabbri editore.

Matteini Chiari Maurizio, Caponi Tiziana (2010). Le preesistenze archeologiche. In Mancini Francesco Federico (a cura di). II Palazzo Donini di Perugia. Perugia: Quattroemme, pp. 37-62.

Mobilità e relative infrastrutture nella citta di Perugia. (1985). Perugia: Comune di Perugia.

Neri Maria Luisa (2009). Alessandro Arienti e l'architettura del palazzo. In Mancini Francesco Federico (a cura di). I/ Palazzo della Provincia di Perugia. Perugia: Quattroemme, pp. 9I-II2.

Quici Fabio (2017). Architecture andVisual Narrative. In Proceedings of International and Interdisciplinary Conference IMMAGINI? $9,1,1082$.

La Rocca Paolina di Perugia. Studi e ricerche. (1992). Perugia: Electa Editori Umbri.

Tarchi Ugo (1942). L'arte del Rinascimento (e secoli posteriori) nell'Umbria e nella Sabina. Milano: Garzanti.

Tomassoni Italo (2003). Beuys a Perugia. Cinisello Balsamo: Silvana editoriale.

Tomassoni Italo (a cura di). (1980). Beuys/Burri. Perugia: Comune di Perugia.

Tosti Mario, Mori Daniela (2009). Il Palazzo della Provincia, il Palazzo del Governo, il Palazzo della Democrazia. Uomini, uso e funzioni di Palazzo Arienti dall'Unità ad oggi. In Mancini Francesco Federico (a cura di). Il Palazzo della Provincia di Perugia. Perugia: Quattroemme, pp. I61-202.

Trinei Marco (20 I6). Fotografie e fotografi di Perugia 1850-19/ 5. Perugia: Futura edizioni.

Proposta di sistemazione della Rocca Paolina. In Belardi Paolo (a cura di). Disegnare Perugia. "Sui tracciati della storia". Rimini: il Cerchio, pp. II5-121.

Sensational Umbria. (20|4). Milano: Sudest57.

Schepers Jörg (a cura di). (1995). Fabrizio Plessi. La rocca elettronica. Videoinstallazioni e disegni. Ponte San Giovanni: Grafiche Benucci.

\section{Autore}

Luca Martini, Accademia di Belle Arti “Pietro Vannucci” di Perugia, lucamartini 1978@gmail.com

Per citare questo capitolo: Martini Luca (2020). Una fortezza papale introversa trasfigura in uno spazio pubblico connesso. L'immagine della Rocca Paolina di Perugia/An introverted papal fortress transfigures into a connected public space. The image of Rocca Paolina in Perugia. In Arena A. Arena M., Brandolino R.G., Colistra D., Ginex G., Mediati D., Nucifora S., Raffa P. (a cura di). Connettere. Un disegno per annodare e tessere. Atti del $42^{\circ}$ Convegno Internazionale dei Docenti delle Discipline della Rappresentazione/Connecting. Drawing for weaving relationships. Proceedings of the 42 th International Conference of Representation Disciplines Teachers. Milano: FrancoAngeli, pp. 3483-3508. 


\title{
An Introverted Papal Fortress Transfigures into a Connected Public Space. The Image of Rocca Paolina in Perugia
}

\author{
Luca Martini
}

Abstract

Graphic representations of the Rocca Paolina of Perugia always appear evocative of the cultural significance that it assumed in the climate of the times in which each image was conceived. In fact, the architectural drawings of Antonio da Sangallo il Giovane, I540, represent a stately palace, which is also an impregnable fortress, while in the seventeenth and eighteenth centuries, when papal domination was complete, the citadel's profile is comparable in size to the urban fabric. On the other hand, in the nineteenth-century perspectives the point of view is lowered thus emphasising the over-sized monumental proportions of the fortress. And just over three centuries after the plans by Sangallo, Alessandro Arienti's project plan of 1870 overturned the role of the fortress by connecting the remains of the ramparts to the new post-unification city centre with a road ramp that sanctioned the end of the impervious nature of the citadel of Perugia and thus began its transformation into a public space. It is in this sense that the architectural drawings of the escalator complex, which in the early nineteen eighties allowed the people of Perugia and tourists to recapture the complex spaces definitively, can be interpreted; these spaces are now celebrated by geolocalised photographs posted on social networks.

The text presents an iconographic study, interpreting the graphic works that have the architecture of Perugia as their subject, from architectural sketches to comic strips including cinematic frames, insofar as these works reveal the roles assumed from time to time by the Paolina macro-structure in the urban context of the Umbrian capital, as they unmask a series of tendentious looks at a piece of architecture that is still enigmatic today.

Keywords

image of the city, Rocca Paolina of Perugia, images.

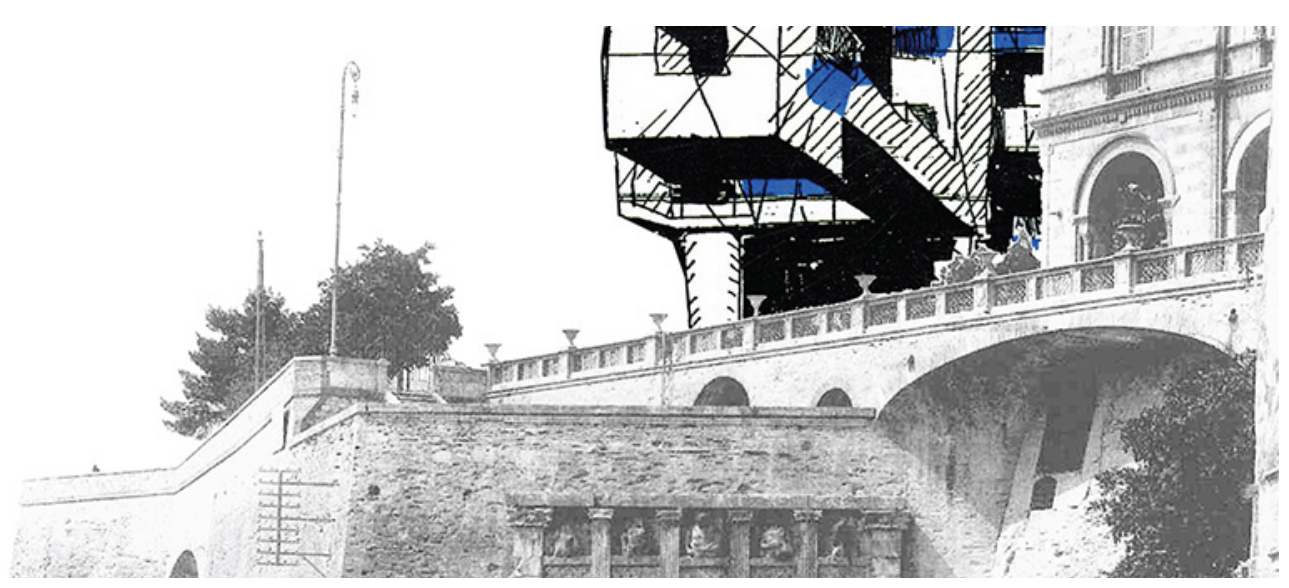


Rocca Paolina in Perugia is an enigmatic piece of architecture. Its elusive character emerges whenever scholars try to understand it, from its initial role as a military fortress to its current function as a public space. To this effect, this research aims to provide a key to interpreting the fortress, based on the graphic representations that have been produced over the centuries and proposing a reading of them that highlights the cultural background of their creators. It will thus attempt a diachronic reading for images of the Pauline architectural palimpsest in the architectural history of the Umbrian capital, through a true path of visual connection [Quici 2017].

The design drawings of the 1540s by Antonio the Younger and his entourage [I] note the Etruscan-Roman fragments of the Umbrian city, clearly revealing Sangallo's intentions for an inclusivist design. However, they do not adequately take into account the papal will, and in fact Pope Paul III overturned their point of view, minimising the role of the defensive stronghold towards the countryside and emphasising the building's military character towards the city [Belardi, Ceccucci 2006; Belardi, Martini 20 I5]. It was built over the residences of the unruly Baglioni family, to such an extent that it covers an entire portion of the city with

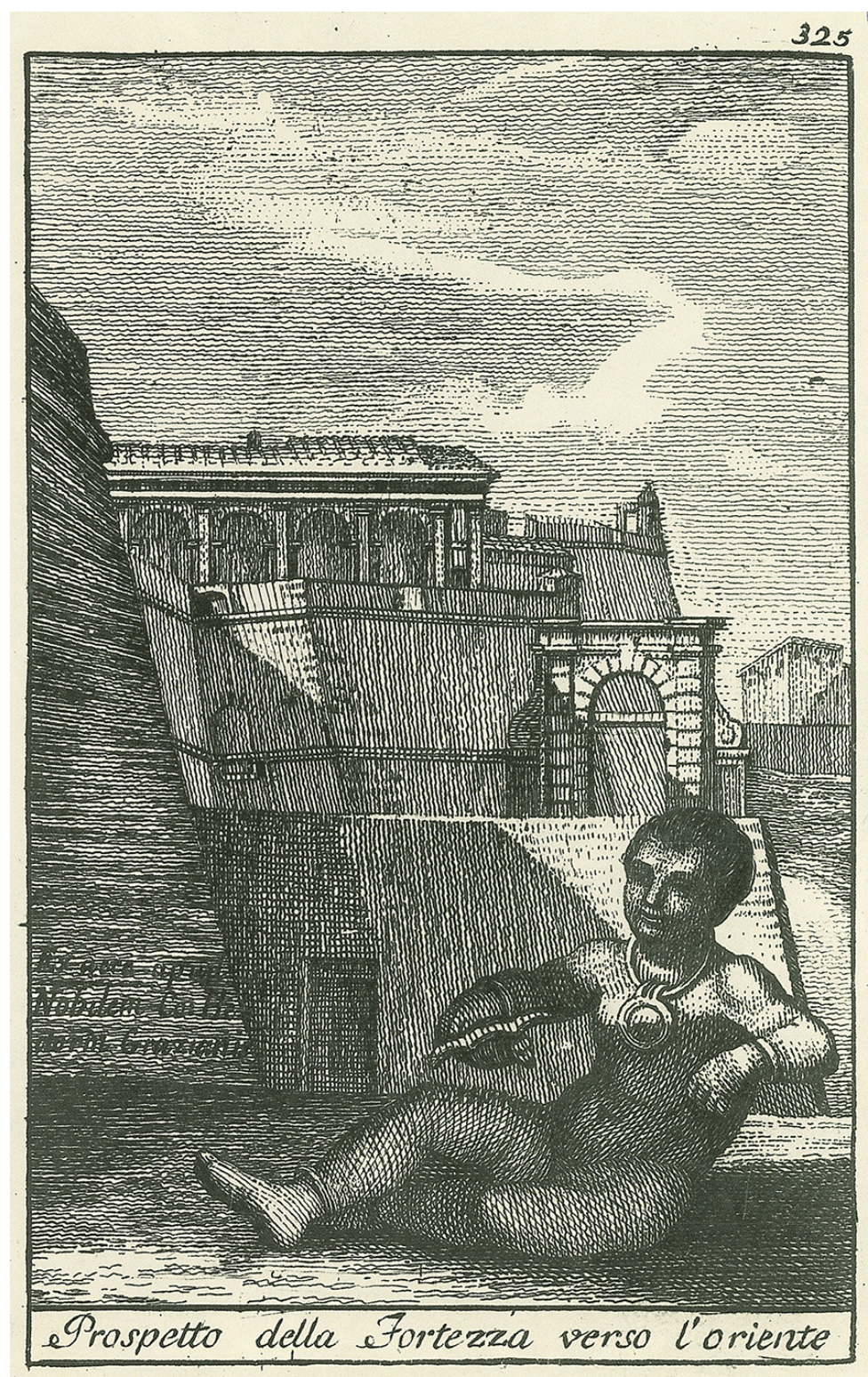


an embankment bordered by defensive walls that transform a medieval city into a dug-in barracks, so the buildings' exterior facades become interior backdrops which decisively subvert the urban image. In this sense, the project drawings describe the fortress according to the intentions of Sangallo, who soon, however, stopped managing the construction site directly [Camerieri, Palombaro 1992, pp. 9- I8; Camerieri, Palombaro 1988, pp. 19-25]. This has led some to suggest that the Pauline project may have been executed by a young Galeazzo Alessi, who received his training in these first Perugian works, which later led him to dedicate himself to Genoa and Milan [Belardi, Martini 2015; Belardi 20I3; Martini 20I6, pp. $16,59,60]$. It is no coincidence that one of the most drawn parts over the centuries was the loggia that Alessi designed as a refined viewpoint, and which, for example, was the subject of a series of 19th-century India ink and watercolour works by students from the Perugia Academy of Fine Arts. These underscored its dual character given by the rigour of the Doric order and the plasticity of the rustic base, rendered in orthogonal projections and frontal perspectives aimed at interpreting the front design [2].

Fig. 2. Giuseppe Rossi, View of Rocca Paolina 1865.

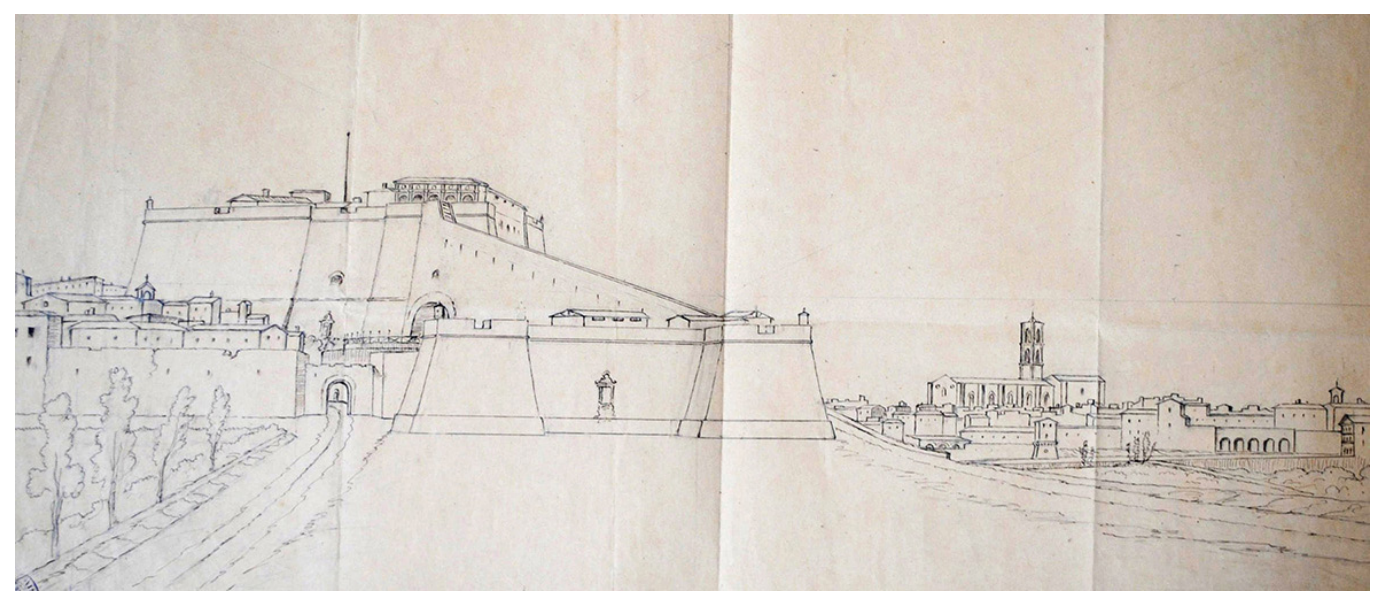

Over the years, Rocca Paolina experienced a bloodless fate, but at the same time symbolised foreign power over the city that submitted to papal rule. And in this sense, in the depictions between the 16th and 18th centuries the bulk of the fortress appears placidly inserted in the city skyline, in views with an observation point very distant from the subject, like bird's-eye views, up to improper points of view, like those of planimetries at an infinite distance. These projections can be seen, for example, in the views by Georgis Hoefnagel (etched in 1574) [3], Cipriano Piccolpasso (I579) [4] and Ignazio Danti (I 58 I-I582) [5], or again in Hoefnagel's perspective etched in the 1640s [6]. Even in the planimetric representations, like the layout by Giovanni Gambini from 1826 [7], although there is a measurable difference in size between the fortress' footprint and that of the other city buildings, often both the graphic choices and the obvious lack of altimetric findings produce a substantial insertion in the urban setting. The same concept was expressed by Baldassarre Orsini's drawing Prospetto della Fortezza verso l'oriente (Perspective drawing of the fortress facing east), etched by Raimondo Faucci in 1784. This portrayed the proleptic relationship between the Alessian loggia, the 17th-century San Carlo Gate and an Etruscan bronze sculpture in the foreground, celebrating the papal city like the ancient one, both remembered over the centuries for their monumental defences [8]. On the other hand, in the decades that followed, the depictions of Rocca Paolina during the 19th century showed a strikingly lowered point of view aimed at amplifying the fortress' oversized dimensions with respect to the size of the city. For example, we see this phenomenon from Orsini's perspective of 1808, but in 
this case the fortress faces the buildings surrounding it, thanks to the point of view set at a height that is in any case greater than a human one [9]. Meanwhile, the fortress appears decidedly more threatening towards the city in Giovanni Monotti's drawing of the 1810s, where the observation point is even lower, even if ultimately the drawing still appears to show a placid view of a provincial town [ 10$]$. This can also be observed in the famous series of oil paintings Giuseppe Rossi produced of the fortress between I860 and 1875, based on a series of earlier drawings [ $\mathrm{I} \mathrm{l}]$. Even if the representation of both forts united by the gallery inserted in the setting unequivocally established their outsized relationship with the inhabited area, presenting that popular feeling that would have led to its physical expulsion from the urban fabric.

At the same time, the construction drawings of the new road routes for the city in transformation testify to the annexation of the fortress area into the urban setting [12]. Subsequently, however, the process that would lead to complete public re-appropriation also involved a traumatic demolition that began in 1848 , following the uprisings that led to the Pope's flight from Rome and the proclamation of the Roman Republic. This event was immortalised by Nicola Benvenuti in his work La demolizione del forte paolino (The demolition of the Pauline fort) from about 1850, in which the ominous mass lies in ruins under the blows of a cheering crowd. The destruction coincides figuratively with the irremediable loss of urban recognisability, which from that time was entrusted to rediscovering the underground spaces used until then for exclusively military purposes, and which would remain practically unacknowledged for over a century [13]. Moreover, while the freedom the Perugians regained was only temporary, since the papal restoration took place in 1849, the fortress' image would no longer be the same. For example, the survey drawings of the remains and the design for a new barracks commissioned from several officers of the papal corps of engineers

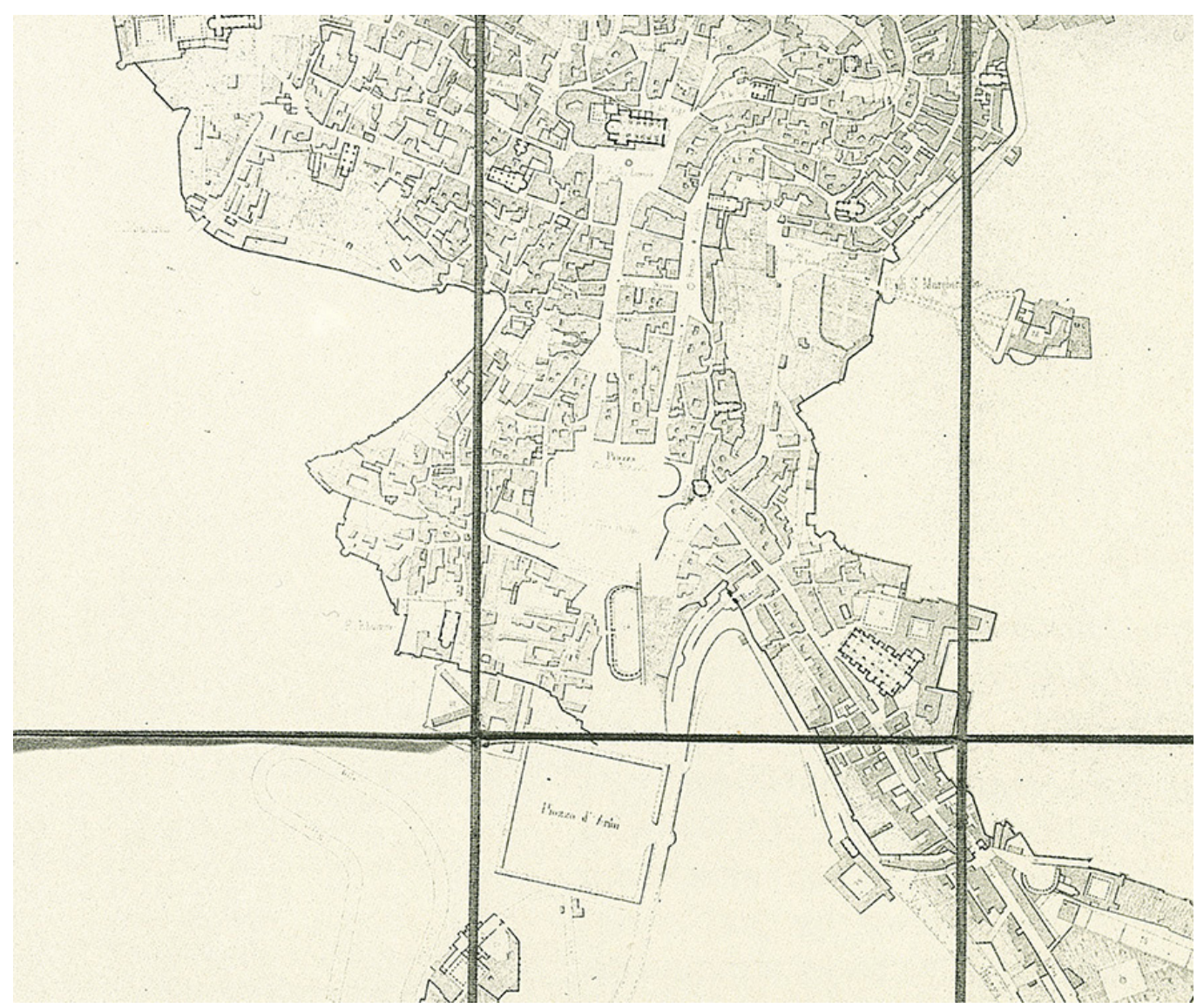


between 1849 and 1856 document the state of ruin of the area of the former Piazza Rivarola [14], corresponding to a photograph from the late 1850s by the photographer Robert Macpherson [15]. This condition can also be seen ina set of drawings and paintings from the mid-19th century by the painter Silvestro Massari, preserved in the Perugia Academy, which also portray the enigmatic Alessian loggia and the chiaroscuro interiors, prefiguring their reconfiguration and reuse, of which unfortunately only the latter would take place [16]. Furthermore, a photo from those years highlights the Marzia Gate incorporated in the Sangallesque walls that had been filled in, to discourage any interest in the underground remains [17]. In fact, shortly thereafter the photos testify to the first surface work in the areas once occupied by the forts, like the Politeama Theatre by Guglielmo Calderini [18]. But at the same time, the topographical plan etched by Giulio Delicati in 1866 shows an empty space where the fortress once stood, reaffirming its state of urban emptiness [19]. In fact, the numerous projects that studied the site near the city centre, though involving illustrious architects (including Nazareno Biscarini and Alessandro Arienti), did not envisage reusing the underground parts, the only surviving elements of the fortified palimpsest, but eliminated the surface ruins and left only the basement spur to the south-east as a sign of them [20]. However, on closer inspection, two drawings by Arienti from 1870 display the relationship of the new provincial administration building with the underlying areas. In particular, the diagram of the foundations and the details of the reinforcements of the supporting walls testify to the physical link between the ancient underground fortress and the new above-ground building [2I]. But above all it was a design plan by Arienti, also from I870, that changed the fortress' role. Here, the remains of the ramparts that guard incredible underground spaces are connected to the new post-unification city centre with a ramp of the new road built at the same time as the public building, which once again highlights the presence of an almost forgotten subterranean city [22]. Although over the years a number of surveys of the underground portion of the fortress were carried out when ownership was transferred between public administrations, the iconographic oblivion continued in the following period, which corresponded to an occasional use of the parts without rubble [23]. In the same way, the early 20th century photos that portrayed the Marzia Gate, while

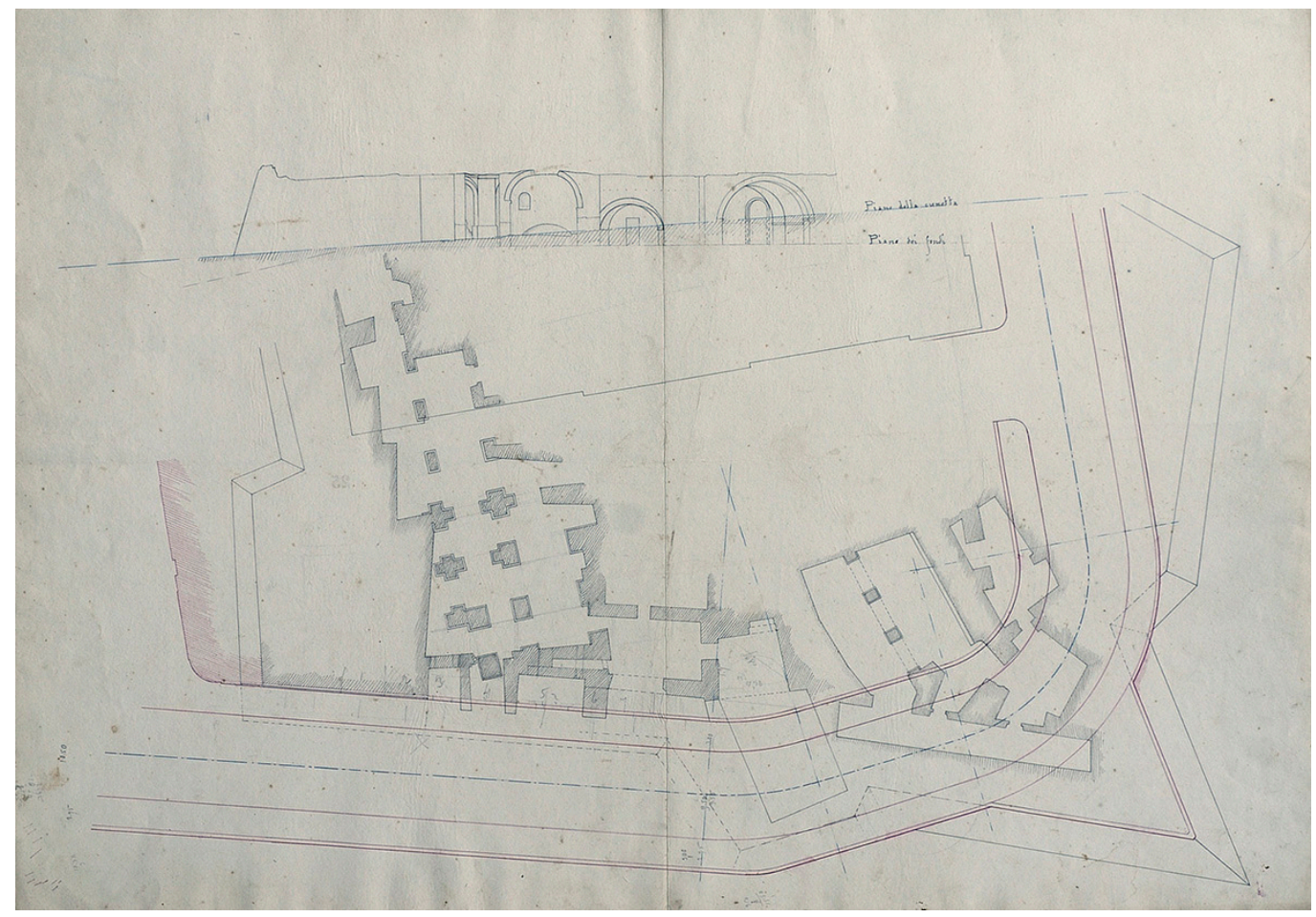


testifying to its reopening, the start of recovery and the condition of urban stratification, implicitly confirm the inaccessibility of the interior [24]. Even if the documents reported that parts of the underground space began to be recovered in the 1930s [Lattaioli 1992, pp. 172-175], it took until 1942 for interest in the research to reawaken through a reconstructive drawing of the Farnese fortress by Ugo Tarchi [25]. In 1943, it was reused as an anti-aircraft shelter for the government building, as emerged from some design documents [26]. In the following years in particular, some areas were used for exhibitions, for example in 1980 for the world-famous initiative that saw participation from the artists Alberto Burri and Joseph Beuys, immortalised by the Rome photographer Lionello Fabbri [27].

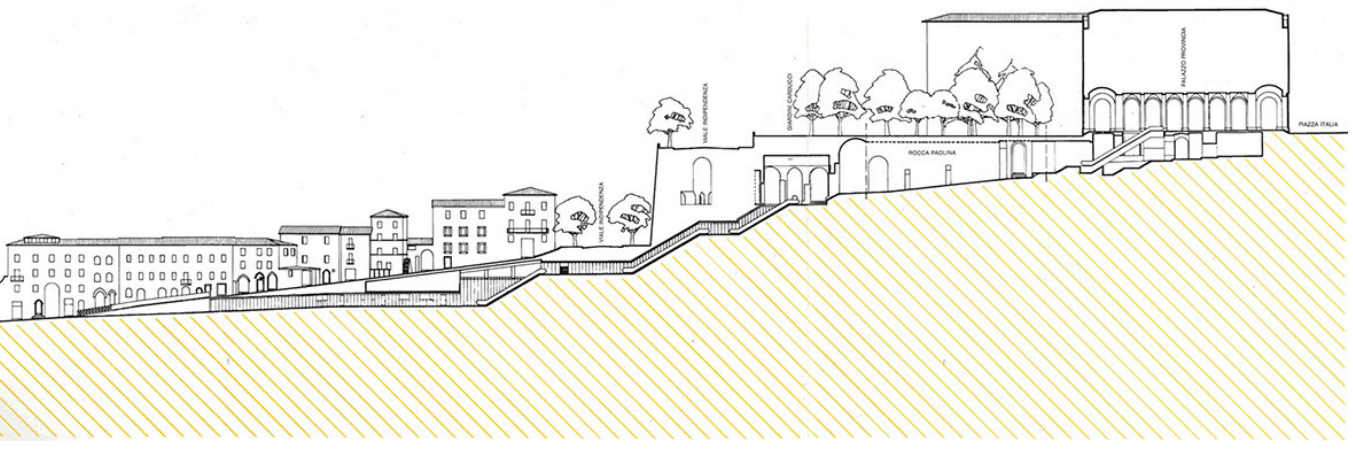

Shortly thereafter, the real coup de théâtre that marked the fortress' public re-appropriation took place in 1983 with the construction of the escalator system that crossed it, connecting the Piazza Partigiani area with the acropolis of the Umbrian capital. This development was represented in the technical drawings of 1979 by the team of the Municipality of Perugia's Technical Office, coordinated by chief engineer Mario Belardi, which marked the definitive re-appropriation of the historic underground spaces. They thus became the setting captured by tourists in selfies posted on social media or in photo spheres on Google Maps [28]. The wave of communications with the fortress as its star was subsequently reinforced by the director Liliana Cavani, who chose it as a medieval setting for scenes in the 1989 feature film Francesco, which cinematically proclaimed its chiaroscuro paths as an emblem of the Middle Ages. In this sense, in those very years, multidisciplinary scientific studies on the fortress' history began to emerge in dedicated publications containing interpretive drawings and reconstructive hypotheses [La Rocca Paolina 1992; Camerieri, Palombaro 1988], culminating in 2009 with the publication of the volume II palazzo della Provincia di Perugia (The palazzo of the Province of Perugia) [Mancini 2009]. The didactic design exercises promoted by Adriana Soletti and Roberto de Rubertis, however, go back to the early 1990s, when on two public occasions they presented drawings prepared by students aimed at examining the area's accessibility, reconnecting it to the urban setting and prefiguring a spatial and technological extension of the escalator system [29]. Likewise, the photos showing the video installations by Fabrizio Plessi in 1995 on the occasion of the exhibition La Rocca Elettronica also connect the fortress' spaces to the contemporary situation [Schepers 1995; Lattaioli, Lattaioli 2004, pp. 108, 109]. In a similar vein are those depicting the City Museum promoted by the Municipality of Perugia and designed by Italo Rota between 1999 and 2003, in which screen-printed glass, captivating graphics and communicative projections were juxtaposed with the centuries-old stone [Belardi, Merli 2009, p. 8 I]. The materials exhibited in that setting, and still visible today, included polymerised plastic models of the Medieval and Renaissance city made by ONEOFF in 2004 using new rapid prototyping technologies, based on digital renditions by Paolo Camerieri and Francesco Miniati. In 2004, with the collaboration of 
Fabio Palombaro, they also animated the fortress' historical events in the video La forma della memoria. La città ritrovata. La costruzione della Rocca Paolina di Perugia (The shape of memory. The rediscovered city. The construction of Rocca Paolina in Perugia), thanks to the reconstructive possibilities of 3D graphics [30]. On the other hand, five years later Daniele Giovagnoni and Marco Vergoni drew and scripted the monument's historical timeline in a comic book that illustrated its latent urban potential in a popular way [Giovagnoni, Vergoni 2009] [3I]. Finally, in 2012 the renowned photographer Steve McCurry portrayed the fortress' spaces for the Sensational Umbria communication campaign promoted by the regional administration, placing them in a figurative relationship with the Umbria Jazz event, which for decades has been rediscovering parts of Perugia's historic centre in a spirit of social and cultural aggregation [Sensational Umbria 20 I4].

To this day the fortress appears as a dreamlike place between past and future, almost eternally 'unfinished' and only partially inserted in Perugia's urban setting, a palimpsest that is in some ways still interrupted [32]. To this effect, Digital Design students from the Degree Course in Building engineering and Architecture at the University of Perugia took part in the danteum. oggi projects in the 2012-20 I3 academic year, creating physical paths of self-knowledge between the underground areas and the 'immensity of the sky', using the languages of architectural composition and representation. For example, the tensions of light project amplified the multi-faceted underground spaces in a stereometric raised garden located iconically above the provincial administration building, connecting them with an ascending ramp that used contemporary digital drawing techniques to reinterpret Dante's worlds and the design experience of Giuseppe Terragni [33]. Finally, the architectural mapping drawn up by Giulia Bazzucchi in the 2016-2017 academic year, for her master's degree thesis in Building engineering and Architecture, reconnects the thread linking the city and the fortress with plans and sections of spaces placed in context for the first time, giving fresh meaning to the Pauline maze in an urban key [Bazzucchi $2016-20$ I7]. In addition, it is no coincidence that it is a 'click of the mouse' drawing that analyses the environments cognitively, since drawing is the speculative and prefigurative tool par excellence. In this sense, the visionary parable of a 'surveyed fortress' that becomes a 'designed fortress' is more desirable than ever, as 'new looks' and 'new images' represent the contemporary city's future [34]. Including in Perugia.

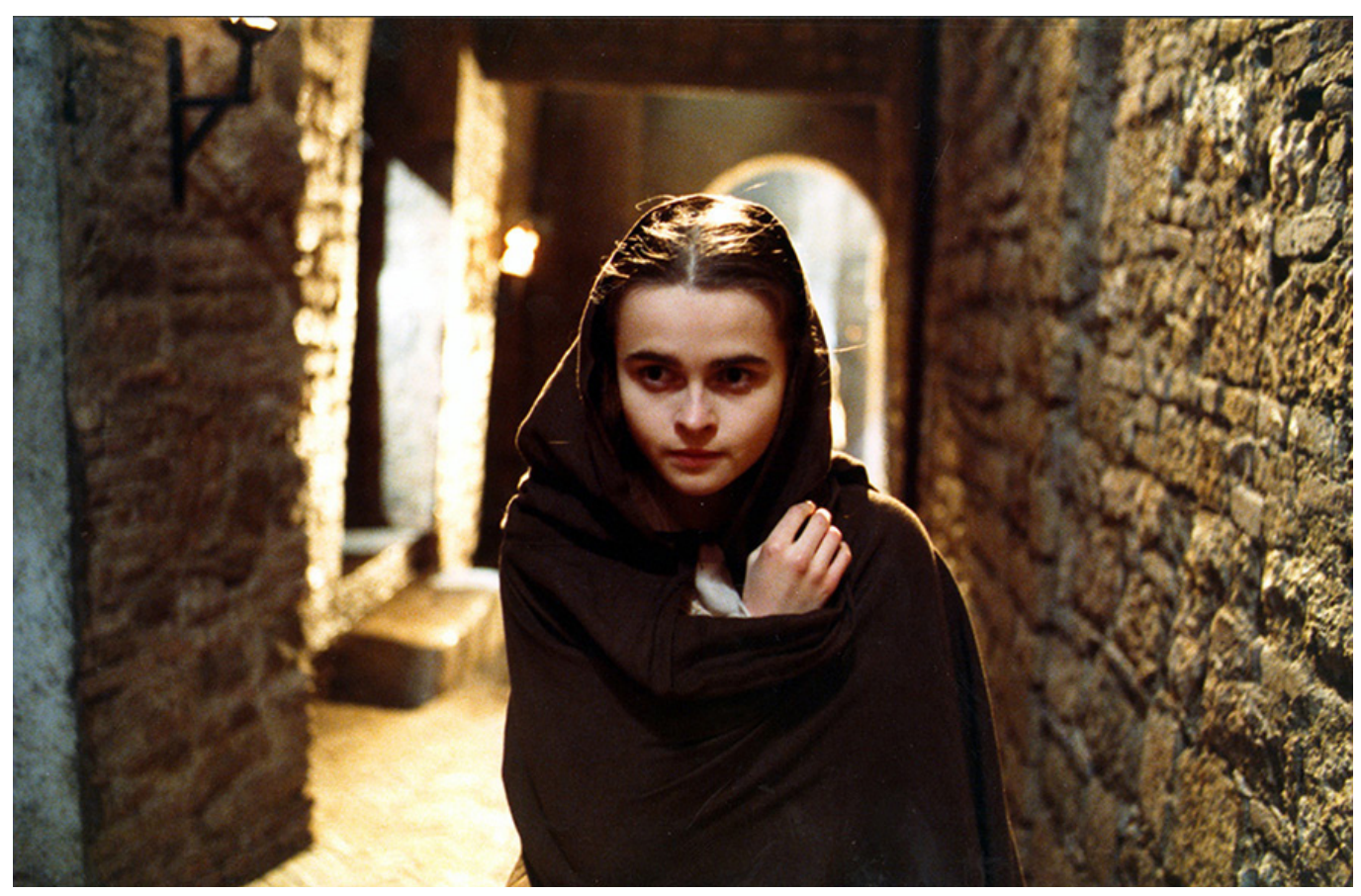




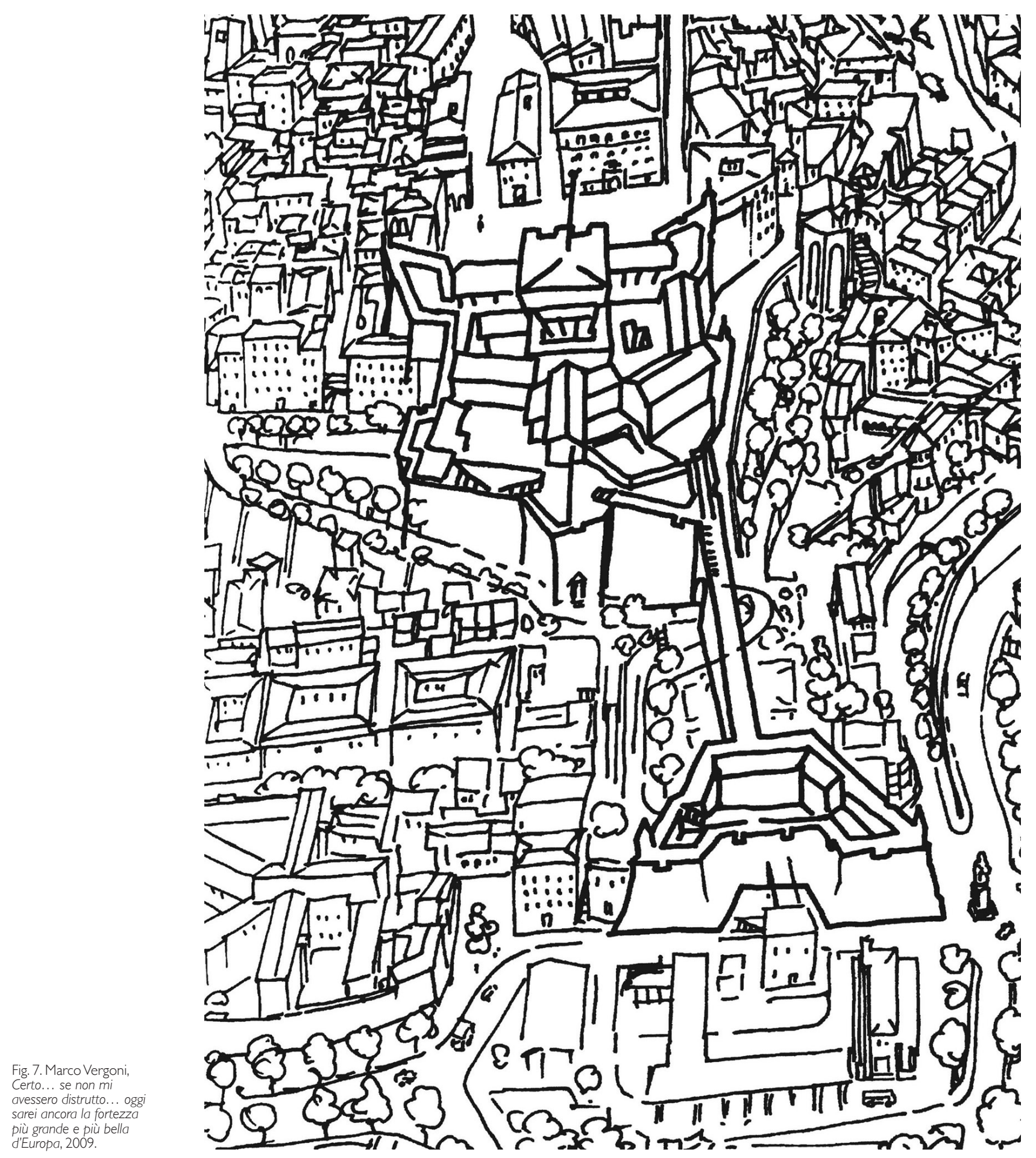



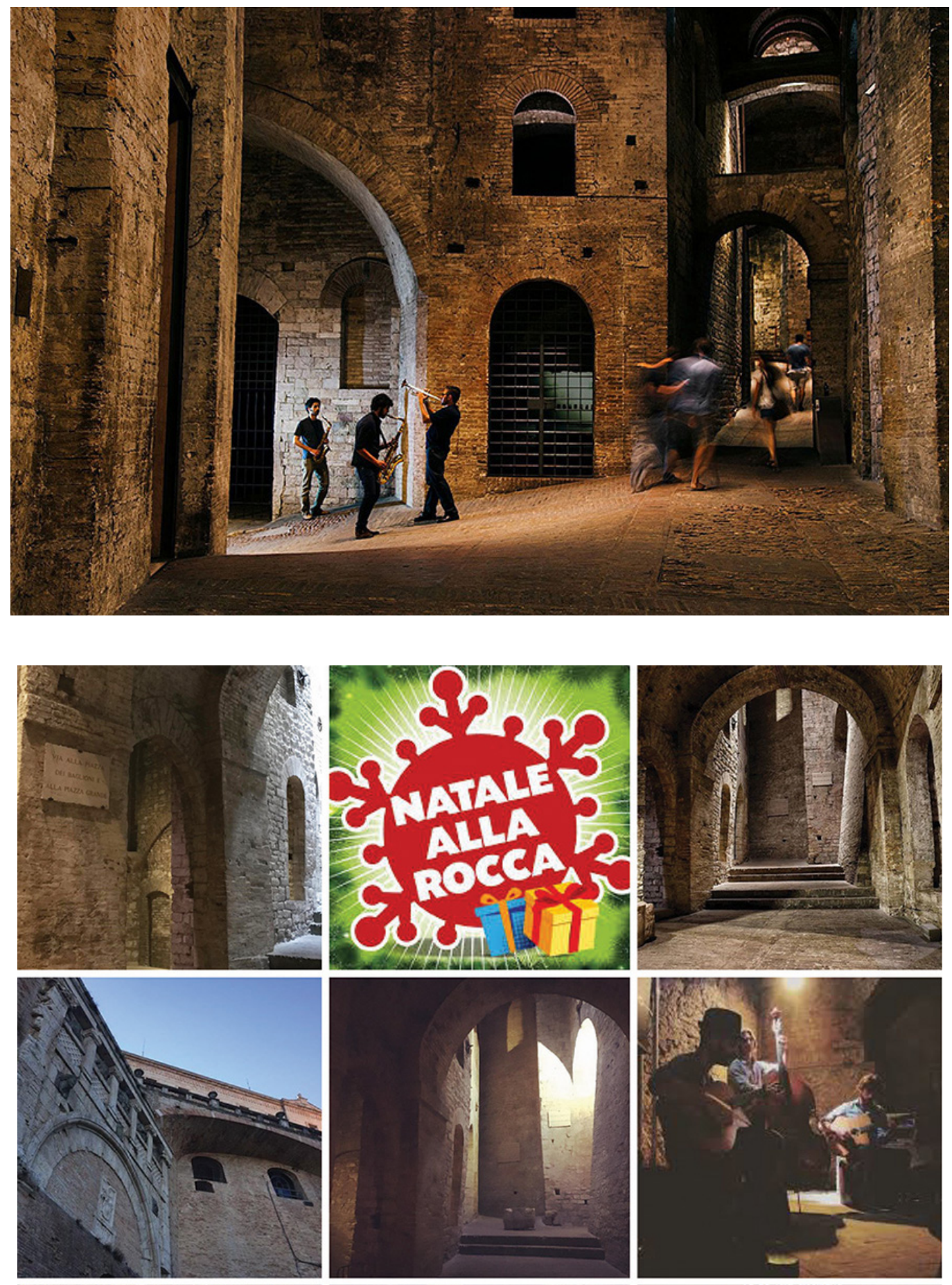

Fig. 9. \#roccapaolina,
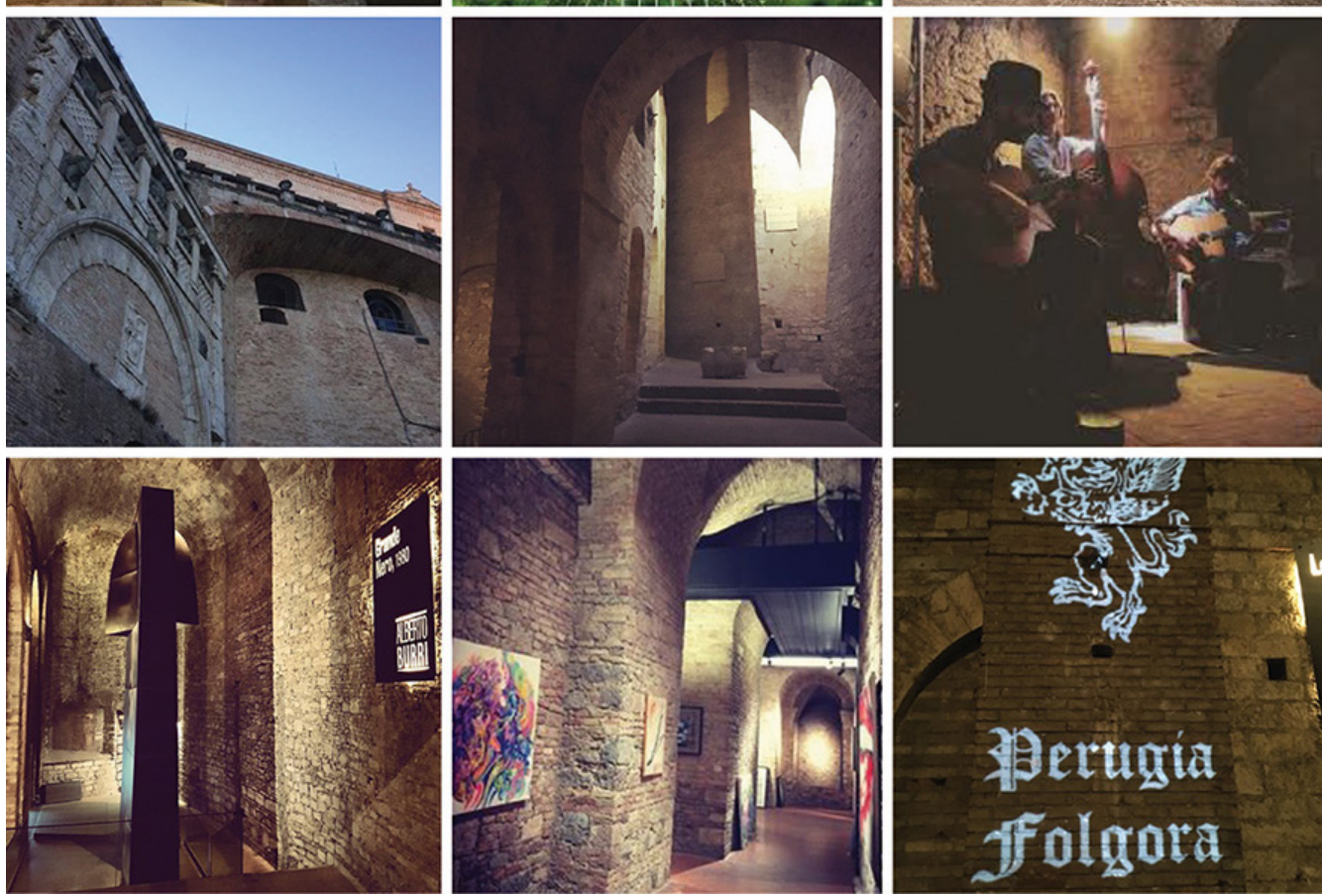
Fig. 10. Luca Martini, Città spaziale. Perugia e locandina dell'iniziativa danteum. oggi, 2013.

Fig. I I. Marta Berrettoni, Elisa Bettollini, Eugenio Bini, Marco Calderini,

Malvina Maria Manfroni,

Simone Moroni, Daniele

Ripa, tensioni di luce, 2013;

Fig. 12. Giulia Bazzucchi, Nuova luce sulla rocca.

Rilievo architettonico della Rocca Paolina di Perugia e progetto illuminotecnico della Casa di Gentile Baglioni, 20 17; section.
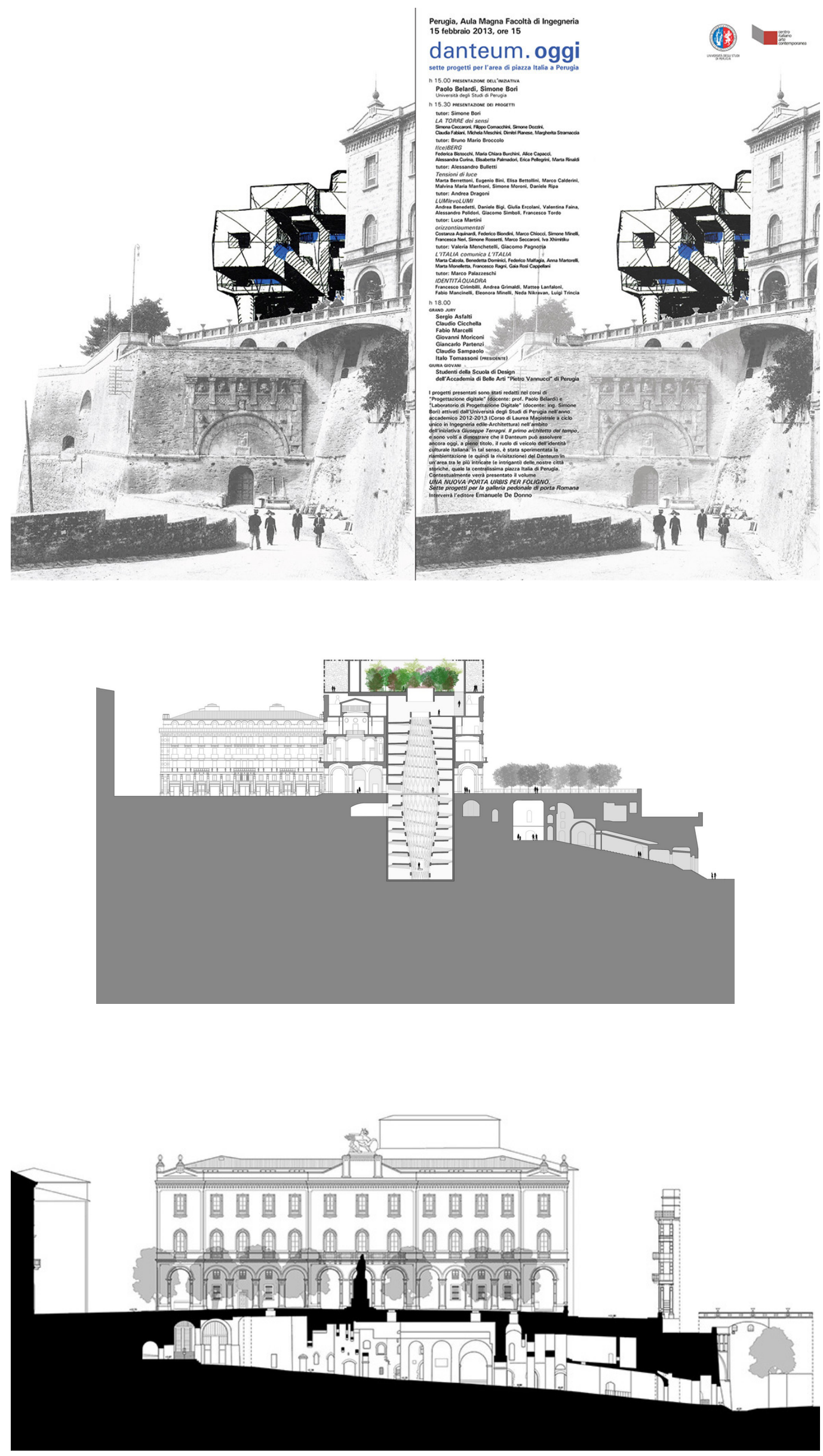


\section{Notes}

[I]The Sangallo drawings are kept in the Department of Prints and Drawings at the Uffizi Gallery in Florence [La Rocca Paolina 1992, pp. 2 I, 23, 28, 29, 33, 47, 5 I, I I0, I I2, I I 5, I I6, I I8; Camerieri, Palombaro 1988, pp. 90, I27- I 50].

[2] The works are kept in the archive of the "Pietro Vannucci" Academy of Fine Arts of Perugia. See: Odoardo Biagini, Pianta e alzato della loggia Alessi (Plan and elevation of the Alessi loggia), I 883; Lorenzo Carloni, Alzato e pianta della loggia dell'Alessi (Elevation and plan of the Alessi loggia), I883. In addition, another drawing is kept in the National Gallery of Umbria, see Stanislao Nicolai, Portico dell'Alessi situato nel Forte (Alessi portico located in the Fort), 1824. See also the design of two fountains in the fortress square, also kept at the Academy of Perugia: Napoleone Cherubini, Pianta Topografica di piazza della fortezza (Topographical Plan of the fortress square), 1834.

[3] Franz Hoogembergh, Perusia. Gratum musis in Tuscia domicilium, I 574 [Cassano 1990, I, pp. 202, 203].

[4] Cipriano Piccolpasso, II primo libro delle Piante et ritratti delle Citta e Terre dell'Umbria (The first book of Plans and portraits of the Cities and Lands of Umbria), I579, f. XXXIII (manuscript 3064 kept at the Augusta Library in Perugia) [Cecchini 1963].

[5] Danti's view is frescoed in the Belvedere gallery in the Vatican Museums: Ignazio Danti, Perusiae Augustae. Corographia, 158I-I 582 [Matteini Chiari, Caponi 2010, p. 38].

[6] Matthäus Merian, Perusia, 1640 [Cassano 1990, II, pp. 220-22 I].

[7] Giuseppe Canacci, Pianta della Città di Perugia (Plan of the City of Perugia), I 826 [Cassano I 990, I, pp. 250-25 I].

[8] Raimondo Faucci, Prospetto della Fortezza verso l'Oriente (Perspective of the Fortress towards the East), I784 [Cassano 1990, II, pp. 14, 15; Belardi 2019].

[9] Raimondo Faucci, Prospetto della Piazza Nuova dinanzi alla Fortezza, con le Fabbriche che sono situate attorno... (Perspective of Piazza Nuova in front of the Fortress, with the Buildings located around...), I 808 [Cassano 1990, II, pp. 40, 4I].

[ I0] Giuseppe Carattoli, Frezzolini, Prospetto della Piazza Napoleone e Fortezza in Perugia (Perspective of Piazza Napoleone and Fortress in Perugia), 1810 [Cassano 1990, II, pp. 68, 69]. See also the perspective by Annibale Angelini: Annibale Angelini, Napoleone Cherubini, Piazza di Minerva una volta Collegio Pio disegnata come se avesse avuto il suo compimento (Piazza di Minerva formerly Collegio Pio, drawn as if completed), I 835 [Cassano 1990, II, pp. 90, 9I].

[I I] Rossi's drawings are kept in the archive of the "Pietro Vannucci" Academy of Fine Arts of Perugia and his paintings at the National Gallery of Umbria. See: Giuseppe Rossi, Veduta del fianco occidentale della Fortezza Paolina (View of the western side of the Paolina Fortress), I 870 [Belardi, Bori, Menchetelli 20 I0, p. 66; Guerrini 1992, pp. I22, I23; Lattaioli 1992, pp. I 38, I39, I45].

[12] For example, the road that connects the area of the so-called Gate of the Three Arches with the current Piazza Italia, designed in 1829 by the pontifical engineer Giovan Battista Cerrini. The related technical drawings are kept in the State Archives of Rome [Lattaioli 1992, pp. I4I-143].

[13] Benvenuti's work is kept in a private collection. See: Nicola Benvenuti, La demolizione del forte paolino (The demolition of the Pauline fort), I 850 [Belardi, Bori, Menchetelli 20 I 0, p. 66]. See also Annibale Brugnoli, Benedetto Baglioni si reca ad abbattere il Forte Paolino (Benedetto Baglioni goes to knock down the Pauline Fort), I 890 [Lattaioli, Lattaioli 2004, p. 77].

[14] For example, the documents prepared by Costantino Forti are kept at the Superintendence of Archaeology, Fine Arts and Landscape of Umbria and the State Archive of Rome [Banti, Ercolani 1992, pp. 195- 198, 202-206, 208-209; Banti, Ercolani 1992, pp. 199-201].

[15] A print of Macpherson's photograph is kept in the library archive of the University of Glasgow. See: Robert Macpherson, Porta etrusca di Perugia (Etruscan gate of Perugia), 1858 [Trinei 20 I6, p. 28].

[16] Silvestro Massari's drawings and paintings are kept in the archive of the "Pietro Vannucci" Academy of Fine Arts of Perugia [Belardi, Merli 2009, p. 58].

[17] The photograph by an unknown photographer is kept in a private collection. View of the walled up Marzia Gate, I865 in Bozzi 1992, p. 226.

[18] Alessandro Baldeschi Eugenj, view of Perugia from the Educatorio Sant'Anna, I865 [Trinei 20 I6, p. 35]; Alessandro Baldeschi Eugenj, view of Perugia from the bell tower of Santa Giuliana, 1870 [Trinei 20 I 6, p. 35]; Cesare Polozzi, view of Perugia from the bell tower of San Pietro, 1880 [Trinei 2016, p. I64]. The photographs are kept in a private collection.

[19] Giulio Delicati, Pianta topografica della città di Perugia (Topographical plan of the city of Perugia), I866 [Cassano I990, I, pp. $274,275]$.

[20] For example, in the project proposal for the public competition of 1863 by Nazareno Biscarini and Americo Calderini, preserved in the archives of the Academy of Perugia, the underground spaces are not involved in the project even though they are shown in the drawings. See: Nazareno Biscarini, Americo Calderini, elevation, 1863 [Neri 2009, p. 95 ].

$[2 \mathrm{I}]$ Alessandro Arienti's design drawings are kept in the archives of the Province of Perugia. See: Alessandro Arienti, foundations layout, 1870 [Neri 2009, p. 105].

[22] Alessandro Arienti, road plan of Viale Indipendenza, I 870 [Belardi, Merli 2009, p. 70].

[23] For example, the surveys of the provincial administrative building are kept in the District Notarial Archive of Perugia: Pianta dei fondi (basement plan), 1874 [Tosti, Mori 2009, p. I62].

[24] Fratelli Alinari, Porta Marzia (Marzia Gate), 1890 [Trinei 2016, p. 204]; Fratelli Alinari, Porta Marzia, 1920 [Bozzi 1992, p.

247]. Both shots are kept in the Alinari archives in Florence.

[25] Ugo Tarchi, Porta Marzia, I 942 [Tarchi I 942, t. LXXX]. 
[26] The 1943 anti-aircraft shelter design is kept in the State Archives of Perugia: Profilo longitudinale del nuovo braccio di galleria (Longitudinal profile of the new gallery branch), 1943 [Tosti, Mori 2009, p. I88].

[27] This encounter also left traces in contemporary Perugia, since the blackboards on which Beuys drew his art lessons are currently exhibited at the civic museum of Palazzo della Penna, while following this initiative Burri's sculpture Grande Nero is still exhibited beside the fortress' escalators [Tomassoni I980; Tomassoni 2003, pp. 22, 25, 38]

[28] Mobilità 1985. See also:Technical Office of the Municipality of Perugia, Percorso pedonale meccanizzato tra il parcheggio pluripiano di Piazza Partigiani e Piazza Italia (Mechanised pedestrian route between the multi-storey car park in Piazza Partigiani and Piazza Italia), 1979 [Belardi, Merli 2009, p. 72; Lattaioli 1992, pp. 176, I77].

[29] See: Disegnare Perugia 1992 and Proposta di sistemazione 1993, pp. I I - 12 I. See also: Roberto de Rubertis, Proposta per la sistemazione di Aliante sul bastione meridionale della Rocca Paolina (Proposal for the arrangement of Aliante on the southern bastion of Rocca Paolina), 1995 [Belardi, Merli 2009, pp. 80, 81]; Roberto de Rubertis, Perugia. Tracciato delle mura etrusche (Perugia. Layout of the Etruscan walls), 1983 [de Rubertis 201 I, p. I7]. Also: Cancellaro 1994-1995.

[30] See the online video animation: <youtube.com/watch?v=LHrxTb IRGas> (26 february 2020).

$[3 \mathrm{I}]$ The one mentioned is not the only time the fortress has been depicted in a comic book story; in 1980, Moreno Chiacchiera represented the fortress under construction in the volume Due o tre cose che sappiamo della nostra città... (Two or three things we know about our city...) [Chiacchiera, Peverini, Todini 2017, p. 49] and in 1983 the French cartoonist Gilles Chaillet drew an external setting of medieval Perugia evidently inspired by the fortress' interiors in the first issue of the Vasco series, L'or et le fer [Chaillet 1983, I].

[32] In November 20 I 4, Rocca Paolina was the focus of the seminar Environments, Rooms and other Sound Spaces. Interscalarity and Multisensoriality in Rocca Paolina in Perugia, scientific director Prof. Alessandra Capanna, as part of a Doctorate in Architecture - Theories and Design at the Department of Architecture and Design at the Sapienza University of Rome. see: Anna Riciputo, Wired Voices | Vocifilate. Lo spazio delle installazioni sonore (The space of sound installations) at: <utopiaspa: <wordpress.com/2017/ I / /05/istallazione-wiredvoices>. In addition, since 2017, Rocca Paolina has been hosting the immersive video installation Perugia Folgora which, though in the limited space of a single room, attempts to poetically reconnect phases of the city's history with the 16th century vaults and walls through projected animated images. The video installation was produced by Daring House under the creative direction of Stefano Casertano and Monica Manganelli.

[33] Teaching professors Paolo Belardi and Simone Bori, tutors Simone Bori, Bruno Mario Broccolo, Alessandro Bulletti, Andrea Dragoni, Luca Martini,Valeria Menchetelli, Giacomo Pagnotta and Marco Palazzeschi. In this context, the tensions of light design concept was drawn up by students Marta Berrettoni, Elisa Bettollini, Eugenio Bini, Marco Calderini, Malvina Maria Manfroni, Simone Moroni and Daniele Ripa. In particular, the poster announcing the public presentation was created by graphically composing a historical photograph representing the Marzia Gate, the Sangallesque walls and the Palazzo della Provincia with the drawing Città spaziale. Shanghai (Spatial city. Shanghai) by Yona Friedman, in 2002 to emphasise the stratified character of the area combined with its potential as an ever-changing urban palimpsest.

[34] These issues were also explored in the public presentation Scientific investigations and project proposals for deeper knowledge of Rocca Paolina given by Paolo Belardi on 12 May 20 17, on the occasion of the initiative Rocca Paolina. A story linked to salt by the Rione di Porta Eburnea Association of Perugia, see: Belardi, Martini, Menchetelli 2020.

\section{References}

Banti Alessandro, Ercolani Riccardo (1992). Le trasformazioni dell'area della fortezza di Perugia nel periodo della formazione dello Stato unitario. In La Rocca Paolina di Perugia. Studi e ricerche. Perugia: Electa Editori Umbri, pp. 193-223.

Bazzucchi Giulia (20 I6-20 I7). Nuova luce sulla rocca. Rilievo architettonico della Rocca Paolina di Perugia e progetto illuminotecnico della Casa di Gentile Baglioni. Tesi di laurea, corso di Laurea Magistrale a ciclo unico in Ingegneria edile-Architettura, relatore prof. Paolo Belardi. Università degli Studi di Perugia.

Belardi Paolo, Martini Luca, Menchetelli Valeria (2020). La rocca Paolina di Perugia. Da baluardo dell'inaccessibilità a landmark dell'accessibilità. In Navarro Palazón Julio, García-Pulido Luis José (ed.). Defensive Architecture of the Mediterranean. Vol. XII. Granada: Universidad de Granada, Universitat Politècnica de València, Patronato de la Alhambra y Generalife, pp. I053- I059.

Belardi Paolo (2019). "Ante litteram" hyper-drawings. Marginal notes on the application of proleptic abstraction in the history of art and architecture. In IMG journal, I (Manifesto), pp. 58-67.

Belardi Paolo (20।3). Sui “disegni regolatori” di Galeazzo Alessi. In L'ingegnere umbro, I, p. I I.

Belardi Paolo, Bori Simone, Menchetelli Valeria (20 I0). II palazzo nell'iconografia storica. II terzo prospetto. In Mancini Francesco Federico (a cura di). Il Palazzo Donini di Perugia. Perugia: Quattroemme, pp. 63-77.

Belardi Paolo, Ceccucci Filippo (2006). Ad coercendam Perusinorum audaciam. Galeazzo Alessi e la trasformazione di Perugia in "città farnesiana": considerazioni e ipotesi. In Robotti Ciro (a cura di). Dai Farnese ai Borbone. Famiglie europee. Costruire Stati. Lecce: Edizioni del Grifo, pp. I33-| 42

Belardi Paolo, Martini Luca (20 I5). The Alessi measure in Perugia. In Disegnarecon, I5, pp. 19. I- I9.22.

Belardi Paolo, Merli Sonia (2009). La piazza. Da spalto fortificato a cerniera immateriale. In Mancini Francesco Federico (a cura di). Il Palazzo della Provincia di Perugia. Perugia: Quattroemme, pp. 53-89.

Bozzi Franco (1992). La distruzione della Rocca. In La Rocca Paolina di Perugia. Studi e ricerche. Perugia: Electa Editori Umbri pp. 225-25I. 
Camerieri Paolo, Palombaro Fabio (1992). La Rocca Paolina: dal "palazzo" alla "cittadella". Dal Sangallo a un "modo architettonico" comune. In La Rocca Paolina di Perugia. Studi e ricerche. Perugia: Electa Editori Umbri, pp. 9-18.

Camerieri Paolo, Palombaro Fabio (1988). La «Rocca Paolina» un falso d'autore. Dal mancato compimento alla radicale alterazione del progetto di Antonio da Sangallo il Giovane per il Forte di S. Cataldo. Perugia: Provincia di Perugia.

Cancellaro Pierpaolo (1994-1995). Rocca Paolina. Un progetto ipogeo per Perugia. Tesi di laurea, corso di Laurea in Architettura, relatore prof. Arrigo Rudi. Istituto Universitario di Architettura di Venezia.

Cassano Francesca Romana (1990). Perugia e il suo territorio. Incisioni dal XV al XIX secolo. Perugia:Volumnia.

Cecchini Giovanni (1963). Perugia del '500 nelle testimonianze di un artista. Cipriano Piccolpasso. Perugia: Sezione arti grafiche Istituto statale d'arte.

Chaillet Gilles (1983). Vasco. L'or et le fer. Une histoire du journal Tintin. Bruxelles, Paris: Editions Du Lombard, 1983.

Chiacchiera Moreno, Peverini Aldo, Todini Antonio (2017). Storia di Perugia. Due o tre cose che sappiamo della nostra città... Perugia: Edizioni Era Nuova.

de Rubertis Roberto (20I I). Rilievi archeologici in Umbria. Perugia, Assisi, Orvieto, Otricoli, Spoleto. Napoli: Edizioni scientifiche e artistiche.

Giovagnoni Daniele, Vergoni Marco (2009). La Rocca Paolina. Autobiografia di una fortezza. Perugia: Era Nuova.

Guerrini Giuseppe (1992). La Rocca Paolina: cittadella come microcosmo guerresco e sede di istituzioni nel secolo della sua costruzione. In La Rocca Paolina di Perugia. Studi e ricerche. Perugia. Electa Editori Umbri, pp. I09- I 25.

Disegnare Perugia. "La piazza scopre la Rocca" (1992). Perugia: Galeno.

Lattaioli Paolo (1992). Effetti indotti dalla costruzione della Rocca Paolina sul tessuto urbanistico di Perugia. In La Rocca Paolina di Perugia. Studi e ricerche. Perugia: Electa Editori Umbri, pp. I33-192.

Lattaioli Paolo, Lattaioli Marta (2004). La Rocca Paolina in Perugia. Visita e storia. Perugia: EFFE Fabrizio Fabbri.

Mancini Francesco Federico (a cura di). (2009). Il Palazzo della Provincia di Perugia. Perugia: Quattroemme.

Martini Luca (2016). Alessi disegnato. Rilievo architettonico delle opere umbre di Galeazzo Alessi. Perugia: Fabrizio Fabbri editore.

Matteini Chiari Maurizio, Caponi Tiziana (2010). Le preesistenze archeologiche. In Mancini Francesco Federico (a cura di). II Palazzo Donini di Perugia. Perugia: Quattroemme, pp. 37-62.

Mobilità e relative infrastrutture nella citta di Perugia. (1985). Perugia: Comune di Perugia.

Neri Maria Luisa (2009). Alessandro Arienti e l'architettura del palazzo. In Mancini Francesco Federico (a cura di). II Palazzo della Provincia di Perugia. Perugia: Quattroemme, pp. 9 I - I I 2.

Quici Fabio (2017). Architecture andVisual Narrative. In Proceedings of International and Interdisciplinary Conference IMMAGINI?, $9,1,1082$

La Rocca Paolina di Perugia. Studi e ricerche. (1992). Perugia: Electa Editori Umbri.

Tarchi Ugo (1942). L'arte del Rinascimento (e secoli posteriori) nell'Umbria e nella Sabina. Milano: Garzanti.

Tomassoni Italo (2003). Beuys a Perugia. Cinisello Balsamo: Silvana editoriale.

Tomassoni Italo (a cura di). (1980). Beuys/Burri. Perugia: Comune di Perugia.

Tosti Mario, Mori Daniela (2009). Il Palazzo della Provincia, il Palazzo del Governo, il Palazzo della Democrazia. Uomini, uso e funzioni di Palazzo Arienti dall'Unità ad oggi. In Mancini Francesco Federico (a cura di). Il Palazzo della Provincia di Perugia. Perugia: Quattroemme, pp. 161-202.

Trinei Marco (2016). Fotografie e fotografi di Perugia 1850-1915. Perugia: Futura edizioni.

Proposta di sistemazione della Rocca Paolina. In Belardi Paolo (a cura di). Disegnare Perugia. "Sui tracciati della storia". Rimini: il Cerchio, pp. II5-121.

Sensational Umbria. (20/4). Milano: Sudest57.

Schepers Jörg (a cura di). (1995). Fabrizio Plessi. La rocca elettronica. Videoinstallazioni e disegni. Ponte San Giovanni: Grafiche Benucci.

\section{Author}

Luca Martini, Accademia di Belle Arti “Pietro Vannucci” di Perugia, lucamartini 1978@gmail.com

To cite this chapter. Martini Luca (2020). Una fortezza papale introversa trasfigura in uno spazio pubblico connesso. L'immagine della Rocca Paolina di Perugia/An introverted papal fortress transfigures into a connected public space. The image of Rocca Paolina in Perugia. In Arena $\mathrm{A}$. Arena M., Brandolino R.G., Colistra D., Ginex G., Mediati D., Nucifora S., Raffa P. (a cura di). Connettere. Un disegno per annodare e tessere. Atti del $42^{\circ}$ Convegno Internazionale dei Docenti delle Discipline della Rappresentazione/Connecting. Drawing for weaving relationships. Proceedings of the 42 th International Conference of Representation Disciplines Teachers. Milano: FrancoAngeli, pp. 3483-3508. 